\title{
Wingless promotes JNK/MMPs positive feedback loop mediate tumour microtubes expansion, glioma progression and neurodegeneration
}

\author{
Marta Portela*, Natasha Fahey-Lozano and Sergio Casas-Tintó* \\ Instituto Cajal-CSIC. Av. del Doctor Arce, 37. 28002. Madrid. Spain \\ *Corresponding authors: scasas@cajal.csic.es
}

\section{m.portela@cajal.csic.es}

\section{Summary}

Glial cells display a network of projections (cytonemes) which mediate cell to cell communication. Under pathological conditions like glioblastoma (GB), cytonemes transform into ultra-long tumour microtubes (TMs). These filopodia infiltrate through the brain, enwrap neurons and deplete wingless $(\mathrm{Wg}) / \mathrm{WNT}$, as a consequence GB progress and neurons undergo synapse loss and degeneration. Thus TMs emerge as a central cellular feature of GB which correlates with a poor prognosis in patients and animal models. Here we describe in a Drosophila model for GB the molecular mechanisms behind TMs production, infiltration and maintenance. Glial cells are initially transformed into malignant GB upon EGFR and PI3K pathways constitutive activation, afterwards GB cells establish a positive feedback loop including Wg signalling, JNK and matrix metalloproteases (MMP). In order, Frizzled1 mediates Wg signalling upregulation which activates JNK in GB. As a consequence, MMPs are upregulated and facilitate TMs infiltration in the brain, hence GB TMs network expands and mediate further wingless depletion to close the loop.

\section{Keywords}

Neuron, Glia, Cancer, wingless, Frizzled, Glioblastoma, Tumor Microtubes, JNK, MMPs, Neurodegeneration. 


\section{Introduction}

Glioblastoma multiforme (GB) is the most frequent and aggressive primary malignant brain tumour with a 3 per 100.000 incidence per year [1]. GB patients' median survival is $12-15$ months, with less than $5 \%$ of survival after 5 years [1-4]. The causes of GB are under debate [2], 5\% of the patients develop GB after a low grade astrocytoma [5] and the most frequent mutations include gain of function of EGFR (97\%) and PI3K/AKT pathways (88\%) [6]. The diagnosis, and therefore the treatment of $\mathrm{GB}$, requires a mutations analysis taking into account the high frequency of clones within the same primary GB [7]. Temozolomide (TMZ) has emerged as an effective treatment for GB however, recent discoveries restrict the use of TMZ in GB patients depending on the methylation status of methylguanine DNA methytransferase (MGMT) [7]. Moreover, among other mutations, Isocitrate dehydrogenase (IDH) define the nature and features of GB [8] together with molecular alterations including $1 \mathrm{p} / 10 \mathrm{q}$ deletions and tumour suppressor protein 53 (TP53) and alpha thalassemia/mental retardation (ATRX) mutations $[8,9]$. This genetic and molecular heterogeneity difficult the diagnosis and treatment of this fatal brain tumours.

The recent discovery of an ultra-long tumour microtubes (TMs) network in GB [10] improves our understanding of GB progression and therapy resistance [11]. TMs are actin-based filopodia which infiltrate through the brain and reach long distances within the brain [10]. TMs are required in GB cells to mediate Wingless(Wg)/WNT signalling imbalance among neurons and GB cells. Wg/WNT signal is favoured in GB cells to promote proliferation, at expenses of the neurons which undergo degeneration and cause lethality $[12,13]$. The central role of TMs in GB biology has emerged as a fundamental mechanism for GB rapid and lethal progression thus; it is an attractive field of study towards potential GB treatments. However, the molecular mechanisms 
underlying the expansion of TMs are not well understood and the signalling pathways mediating TM infiltration are still unknown.

Matrix metalloproteases (MMPs) are a family of endopeptidases capable of degrading the extracellular matrix (ECM). Members of the MMP family include the "classical" MMPs, the membrane-bound MMPs (MT-MMPs), the ADAMs (a disintegrin and metalloproteinase; adamlysins) and the ADAMTS (a disintegrin and metalloproteinase with thrombospondin motif). There are more than 20 members in the MMP and ADAMTS family including the collagenases, gelatinases, stromelysins, some elastases and aggrecanases [14]. The vertebrate MMPs have overlapping substrates, they exhibit genetic redundancy and compensation, and pharmacological inhibitors are non-specific. In contrast, there are only two MMP genes in Drosophila, MMP1 and MMP2, categorized by their pericellular localization, as MMP1 is secreted and MMP2 is membraneanchored, suggesting that protein localization was the critical distinction in this small MMP family. Recent reports propose that products of both genes are found at the cell surface and released into media and that GPI-anchored MMPs promote cell adhesion when they are rendered inactive. Moreover, the two MMPs cleave different substrates, suggesting that this is the important distinction within this smallest MMP family [15]. MMPs are upregulated in a number of tumours, including gliomas. MMPs upregulation in $\mathrm{GB}$ is associated with the diffuse infiltrative growth and have been proposed to play a role in glioma cell migration and infiltration $[16,17]$ reviewed in [18]. In consequence, MMPs upregulation in GB is an indicator of poor prognosis [19] and the study of the mechanisms mediated by MMPs is relevant for the biology of GB, and cancer in general.

The Jun-N-terminal Kinase (JNK) pathway has been associated to glial proliferation. GB cells normally activate JNK pathway to maintain the stem-like GB cells, which has become a pharmacological target for the treatment of GB [20]. Moreover, the JNK pathway is the main regulator of MMPs expression and cell motility in different organisms and tissues [21-25]. 
In this study, we have used the Drosophila melanogaster model of glioma [26] to demonstrate that GB cells activate the JNK pathway and upregulate MMPs (MMP1 and 2) expression. MMPs contribute to TMs expansion through the brain and facilitate Frizzled1-mediated Wg/WNT signalling in GB cells. To complete the positive feedback loop, Wg/WNT signalling mediates JNK activation in GB cells to continue with the process. We postulate that the founder mutations in GB (PI3K and EGFR) initiate the process with the expansion of the TMs; afterwards, the system self-perpetuates (TMsFz1/Wg-JNK-MMPs-TMs) to facilitate GB progression and infiltration in the brain.

\section{RESULTS}

\section{MMPs are upregulated in GB}

GB cells tumour microtubes (TMs) infiltrate the brain and contribute to tumour progression and associated neurodegeneration [10, 13, 27]. TMs are actin-based structures similar to cytonemes $[10,13,28]$ which mediate the exchange of signalling molecules among cells that contribute to GB advancement [13]. The mechanisms mediating TMs formation and progression are not well understood, we propose that TMs infiltration through the brain extracellular matrix $(E C M)$ is mediated by Matrix Metalloproteases (MMPs) activity. To determine if MMPs are expressed in GB, we used specific antibodies and reporters to detect Drosophila MMP1 or MMP2 expression. Confocal images show larvae brains stained with anti-MMP1 (Figure 1A-B", E, F) or anti-MMP2 (Figure S1A-B", E and a GFP reporter shown in F) signal increase in GB compared to control glial cells or to neighbouring neurons. To visualize the cytonemes (WT brain) or the TMs (GB samples) we use the specific marker inog-RFP. MMPs proteins colocalize with TMs and are preferentially accumulated in the limiting region of GB and healthy brain tissue (Figure 1B-B", S1B-B" and S1F-F'). To determine the hierarchy of events between TMs formation and MMPs expression, we attenuated 
Gap43 expression in GB cells to prevent TMs formation and stained for MMPs. The results show that TMs do not expand upon Gap43 RNAi expression and MMP1 and MMP2 protein levels are comparable to control levels (Figure 1C-C", E, F and S1C-C", E). Moreover, we reported recently that GB expansion requires Wg signaling, GB cells vampirize $\mathrm{Wg}$ from neighbouring neurons via Frizzled1 receptor (Fz1) accumulation in TMs. To determine Wg pathway contribution to MMP1/2 expression, we silenced $F z 1$ in GB to reduce Wg-pathway signaling and stained for MMPs. As previously reported, GB cells do not over proliferate; in addition MMP1 and MMP2 are not overexpressed upon Fz1 RNAi (Figure 1D-D", E, F and S1D-D", E). All together the results suggest that MMP1 and MMP2 expression depend on TM network formation and Fz1-mediated Wg signalling pathway. These results indicate that GB cells form a TM network which facilitates Wg signalling and then, upregulate MMPs to mediate infiltration of glioma cells in the brain.

\section{PI3K or EGFR individually do not stimulate MMPs expression}

To determine the epistatic relations behind MMPs expression, we studied single gene modifications related to GB and the effect on MMPs. We expressed the constitutively active forms of PI3K (dp110) and EGFR (TOR-DER ${ }^{\mathrm{CA}}$ ) in glial cells marked with ihogRFP. In both cases we did not observe an upregulation of MMP1 or the formation of TMs (Figure S2A-B" and D). PI3K and EGFR pathways converge in dMyc expression [5], thus we tested $d M y c$ upregulation in glial cells as a candidate to upregulate MMPs and form a TMs network. However, we did not observe any significant change in MMPs expression or TM expansion upon $d M y c$ upregulation (Figure S2C-C" and D). Taking these results together, even though Myc is a convergent point of EGFR and PI3K pathways, it is not sufficient to reproduce the features of glioma. These results suggest that both PI3K and EGFR together are necessary to activate a downstream pathway responsible for the expansion of glial projections and MMP1 accumulation in glial transformed cells, but Myc expression is not the cause of these phenotypes. 
The results show that Fz1 is necessary to upregulate MMPs (Figure 1D-D" and S1D$\left.D^{\prime \prime}\right)$ upon GB induction. To conclude if Fz1 is sufficient to trigger MMP upregulation we overexpressed Fz1 in WT glial cells (Figure 2A-E and S1G-I). The results show MMP1 and MMP2 upregulation in glial cells upon Fz1 overexpression as compared to control samples (Figure 2A-E and S1G-I). These results suggest that $\mathrm{Wg}$ pathway is necessary and sufficient to activate MMP expression in glial cells.

\section{JNK activation in glioma}

JNK is upregulated in a number of tumours including GB and it has been related to glioma malignancy [29-32]. Moreover, JNK is a target for specific drugs in combination with temozolomide treatments as it was proven to play a central role in GB progression [20, 33-35]. However, little is known about the molecular mechanisms underlying JNK activation in glioma cells and the functional consequences for GB progression.

To confirm JNK pathway activation in GB cells, we used two independent reporters. pucLacZ monitors the transcriptional activation of the downstream JNK target puckered [36, 37] in addition, Tre-GFP confer transcriptional activation in response to JNK signalling [38-40]. GB cells show a progressive upregulation of puc-LacZ (Figure 3A-D) and TreGFP reporters (Figure 3E-F, J) compared to control samples, indicating that JNK pathway is activated in GB cells. Moreover, to determine if JNK activation depends on TMs formation or Wg pathway activity, we silenced Gap43 or Fz1 respectively and monitored Tre-GFP and quantified the number of Repo+ cells in GB brains (Figure 3GJ). The results show that JNK pathway depends on the formation of the TMs network as well as the presence of $\mathrm{Fz1}$ receptor in GB cells.

\section{Grindelwald receptor mediates GB progression}


Next we decipher the molecular basis of JNK pathway activation in GB cells. eiger (egr) is the ligand for JNK-grnd pathway, to determine the localization of we used a egr-GFP protein fusion and monitored GFP signal in confocal images. The results show that GFP signal is localized in the neurons that contact with glial cells and the egr-GFP signal is higher in GB samples (Figure S3A-E). Next we monitored the expression pattern of the JNK receptor Grindelwald (grnd), we used a specific antibody in GB and control samples (Figure S3F-H). The results show that grnd protein accumulation in glial membranes is increased in GB brains. Taking these data together, GB cells increase the amount of grnd and egr protein is upregulated in the tissues surrounding GB cells.

To study the contribution of grnd to GB progression, we attenuated grnd expression with a UAS-grnd RNAi expressed in glial cells under the control of repo-Gal4. The results show that grnd knockdown does not reduce the number of glial cells during development (Figure 4A, C, G) but prevents the increase of glial cells upon GB induction (Figure 4B, D, G). To further validate the results, we analysed the number of glial cells in a mutant background for grnd (see materials and methods). The results reproduced the previous observations, the number of glial cells was not affected during development but in the case of GB brains, glial cell number was not increased (Figure 4E-G). Thus, grnd receptor is dispensable for glial development and necessary in GB cells for tumour advance.

To further validate the contribution of JNK pathway in GB progression, we modified JNK pathway expressing a dominant negative form of the effector Bsk (Bsk $\left.{ }^{D N}\right)$ in glial cells, or inducing GB in a egr mutant background (egr-/-), or expressing the external domain of grnd which causes a dominant negative effect (grnd extra) in GB cells. In all three situations, JNK pathway disruption in GB cells prevent the increase of glial cells number as compared to controls (Figure S4). Moreover, these modifications also cause a reduction in the number of wt glial cells compared to controls suggesting that JNK signalling pathway is required for normal glial development and GB progression. 


\section{grnd mediates Wg pathway activation in GB}

Wg pathway activity in glial cells is necessary for GB development, as previously described [1]. GB cells accumulate Fz1 receptors which mediate Wg signalling in the tumour at expenses of neuronal depletion of $\mathrm{Wg}$ and, as a consequence, results in neurodegeneration. This accumulation of Fz1 in TMs depends on Gap43. To establish the relation between JNK activation in GB and Wg pathway, we analysed the contribution of grnd in Fz1 distribution (Figure 5), and the activity of Wg pathway (armadillo) in GB brains upon grnd depletion (Figure 6).

Fz1 is homogeneously distributed through the brain under normal conditions (Figure 5A$\left.A^{\prime \prime}, G\right)$ but, under GB conditions, Fz1 is accumulated in the TMs surrounding the neurons (Figure 5B-B", G). The knockdown of grnd expression in glial cells (Figure 5C-C", G) does not affect Fz1 distribution and in GB cells (Figure 5D-D”, G), grnd RNAi restores Fz1 distribution to a normal situation. To further confirm the effect of grnd knockdown on glia and GB cells, we performed identical experiments in a grnd mutant background. Again Fz1 distribution requires grnd expression in GB cells to accumulate around neurons (Figure 5E-G). In addition, we blocked JNK pathway downstream grnd with a dominant negative form of Bsk (Bsk ${ }^{\mathrm{DN}}$ ) [41] in GB cells (Figure S5). The results show that JNK pathway is necessary to localize Fz1 in the TMs and promote GB progression

Next we studied the activation of Wg signalling pathway in glia and GB cells. Armadillo (Arm) is the orthologue of mammalian $\beta$-catenin which translocate to the nuclei to activate transcription of target genes upon $\mathrm{Wg}-\mathrm{Fz} 1$ signalling $[13,42]$. We used an specific antibody (Cyt-Arm) which detects the inactive form of Arm [13, 42] to monitor the activity of the pathway. Control brains show homogeneous signal for Cyt-Arm (Figure $6 \mathrm{~A}-\mathrm{A}^{\prime \prime}, \mathrm{G}$ ) but, as a consequence of Fz1 distribution, GB cells activate $\mathrm{Wg}$ pathway as represented by the reduction of Cyt-Arm signal (Figure 6B'B", G). grnd RNAi or grnd mutant background prevent the imbalance of Wg signalling among GB cells and neurons 
and restores $\mathrm{Wg}$ signalling to a control situation (Figure 6C-G), similar to previous results obtained for Fz1 distribution,

In addition, we analysed the contribution of JNK pathway to the progression of TMs in GB. We used the reporter ihog-RFP to visualize cytonemes in wt glial cells and TMs in GB. The confocal images show that upon downregulation of grnd expression or the inhibition of JNK pathway by $B s k^{D N}$ in GB cells, the formation of TMs necessary for GB development does not occur (Figure 5 and Figure 6). These results suggest that JNK pathway activation mediated by the receptor grnd is a requirement for TMs formation. As a consequence of the prevention of TMs network development, GB cells do not localize Fz1 in the areas of contact with neurons and cannot mediate $\mathrm{Wg}$ depletion. This leads to a reduction in the proliferation of GB cells. Thus the results indicate that grnd activity mediates JNK signalling to form the TMs network in GB necessary to facilitate tumour progression.

\section{JNK triggers MMPs expression in GB}

The mechanisms for TMs progression and GB cells infiltration through the brain are not well understood. GB cells project TMs which cross the extracellular matrix (ECM) and break in the brain to reach territories distant from the original GB site. We propose that JNK activity in GB mediate the production of matrix metalloproteinases (MMPs) to facilitate ECM digestion and, as a consequence, permit TMs infiltration through the brain.

To validate this hypothesis, we first determined if MMPs expression in GB (Figure 1, Figure $\left.7 A-B^{\prime \prime}\right)$ depends on grnd and JNK pathway signalling. We generated a GB and silenced JNK pathway with Bsk ${ }^{\mathrm{DN}}$ (Figure $7 \mathrm{C}-\mathrm{C}^{\prime \prime}$ ) or silenced grnd expression (Figure 7D-E'). The quantification of MMP1 signal showed that GB upregulation of MMP1 is prevented after JNK pathway silencing (Figure 7F). As occurs in epithelial cells, MMP1 is a target of JNK pathway also in GB cells. 


\section{TMs expansion requires MMPs}

Matrix metalloproteinases (MMPs) are extracellular enzymes responsible for the degradation of the extracellular matrix; cancer cells produce MMPs to facilitate tumour progression and invasiveness. MMPs are upregulated in human GBM cell-lines and biopsies as compared with low-grade astrocytoma (LGA) and normal brain samples [43, 44]. In particular, among the 23 MMPs present in humans, MMP9, MMP2 and MMP14 are directly implicated in growth and invasion of GB cells [45].

WNT induces MMPs expression during development and cancer [46-50] associated to cell migration and metastasis. Specifically in human GBs, MMP2 expression and their infiltrative properties correlate with Wnt5 $[51,52]$ and MMP9 is upregulated upon EGFR activity [53]. To elucidate if MMP expression is related to the development of the network as a mechanism to cooperate in tumour infiltration, we analyzed MMPs expression in Drosophila gliomas. There are two orthologues to human MMPs in Drosophila, MMP1 and MMP2.

We have determined that MMP1 is upregulated in glioma tissue induced by Fz1 receptor (Wg pathway) accumulation in TMs (Figure 1) and JNK activation (Figure 7).

To determine the contribution of MMPs to Fz1 receptor accumulation and $\mathrm{Wg}$ vampirization [13] we carried out functional studies. But first, UAS-MMP1 and UASMMP2 RNAi tools [54] were validated in epithelial tissues (Figure S6). MMP1 or MMP2 RNAi were expressed in GFP-marked cells (see material and methods) and stained with specific MMP1 or MMP2 antibodies. Both RNAi tools reduced significantly the signal for MMP1 or MMP2 staining.

Afterwards we silenced MMP1 or MMP2 in GB and stained the brain samples for Fz1 and Wg (Figure 8A-G S7A-G). Confocal images from larval brains show that Fz1 
distribution in control brains (Figure 8A-A") and GB (Figure 8B-B"). The results show that MMP1 or MMP2 RNAi does not disrupt Fz1 brain distribution (Figure 8C-C", E-E", G) nor glial cell cytoneme network (Figure $8 \mathrm{H}$ ). In line with previous reports [13] Fz1 is accumulated in GB cells membranes and this is halted by MMP1 or MMP2 knockdown (Figure 8D-D", F-F", G). Moreover MMP1 or MMP2 interference prevents TMs network formation and expansion in GB (Figure 8D', $F^{\prime}$ and $H$ ).

In addition, $\mathrm{Wg}$ accumulation in $\mathrm{GB}$ membranes (Figure $\mathrm{S} 7 \mathrm{~B}, \mathrm{D}, \mathrm{F}$, and $\mathrm{G}$ ) is prevented by MMP1 or MMP2 attenuation. Moreover, MMP1 or MMP2 RNAi does not affect Wg distribution in control samples (Figure S7A, C, E, and G). Next, to determine the contribution of MMP1 and MMP2 expression in GB to Wg signalling we downregulated MMP1 or MMP2 in GB samples using a Repo-Gal4 driver and a UAS-ihog-RFP to mark the glial network and stained for Cyt-Arm. Control brains show homogeneous signal for Cyt-Arm (Figure S7H-H"', N) but, as a consequence of Fz1 distribution, GB cells activate Wg pathway as represented by the reduction of Cyt-Arm signal (Figure S7I-I", N). Similar to previous results obtained for Fz1 distribution, MMP1 or MMP2 RNAi prevent the imbalance of Wg signalling among GB cells and neurons and restores Wg signalling to a control situation (Figure S7J-N), similar to previous results obtained for Fz1 distribution.

To determine the contribution of MMPs to the lethality caused by GB, we downregulated MMP1 or MMP2 in GB samples and analyzed survival. To avoid developmental defects caused by MMP1 or MMP2 attenuation, we did the survival experiments after metamorphosis, in adults. We used the Gal80 ${ }^{\mathrm{TS}}$ system to silence the binary expression system UAS/Gal4 during development, and switched it on 4 days after eclosion. Glioma lethality was rescued by MMP1 or MMP2 down regulation (Figure 8I) suggesting that MMPs are required for GB lethality. All the data together suggest that MMPs facilitate infiltration and tumour invasiveness in the brain, mediate $\mathrm{Wg}$ pathway imbalance, GB cells increase and TMs projection which induce premature death. 
GB causes neurodegeneration and neurological symptoms in patients [55]. We have previously described that in GB brains TMs expansion and therefore the accumulation of $\mathrm{Fz} 1$ in the projections is having an effect on the neighbouring neurons that accumulate inactive Arm [13]. Moreover, it has been reported that reduction in $\mathrm{Wg}$ signalling causes reduction of synapse number, which is one of the initial events in neurodegeneration [5659]. We wondered if MMPs role in GB would reduce the synapse number in our animal model. We specifically inhibited MMP1 or MMP2 in GB and quantified the number of active zones in neuromuscular junctions. The results showed a significant reduction in the number of synapses in the neurons upon GB induction compared with the control (Figure 9A-B). However, upon MMP1 or MMP2 knockdown in GB, neurons are protected and the number of active zones is rescued (Figure 9D, F, G).

Taking all these data together, we showed that MMPs mediate GB progression and neurodegeneration. Further, MMPs silencing in GB cells is sufficient to rescue life spam in adult flies. These results bring a novel positive feedback loop among Wg pathway, JNK and MMPs which contribute to GB development (Figure 9H), thus opening novel avenues for GB adaptation mechanisms and potential pharmacological targets.

\section{Discussion}

Activating mutations for EGFR and PI3K pathways are the most frequent initial signals in GB. However, the attempts to treat GB reducing the activation of these pathways have so far been limited by acquired drug resistance. The current tendencies suggest that a multiple approach is required to obtain a more positive result [60-64]. GB cells show a high mutation rate and usually present more than two sub-clones within the same patient and from the same primary tumour $[65,66]$.

Here we describe that JNK signalling regulates MMPs expression in GB, which is required for TMs network formation and infiltration. As a consequence, $\mathrm{Wg}$ pathway 
responds to JNK and TMs expansion, these three events conform a regulatory positive feedback loop in GB progression (Figure $9 \mathrm{H}$ ). Grnd is the JNK receptor upregulated in GB cells, it binds to the ligand egr and activates JNK pathway. GFP fusion protein showed that egr is produced in the surrounding neurons and accumulated in the border of GB and healthy neuronal tissue. Therefore, here is another example of neuron-glia molecular interaction which mediates the physiological status of the brain and the evolution of GB. It is of interest to unravel the regulatory mechanisms that mediate egr expression and secretion in neurons as a potential modulator for brain tumour advance.

Frizzled receptors mediate matrix metalloproteinase (MMP) 2 and MMP9 expression in different scenarios. Blocking WNT signaling, or MMP activity, reduces T cell migration through the basement membrane in vitro and into inflamed skin in vivo. MMP promoters respond to WNT signalling through tandem TCF sites and mediate T cell extravasation [67]. MMP9 is a key molecular effector, downstream of HIF-1 $\alpha$ and WNT activation, responsible for increased rates of neural stem cells proliferation and migration in hypoxia [68]. In addition, Mmp7 (also known as matrilysin) is a target gene of the Wnt signalling pathway in lung epithelial cells and is known to be a key mediator of pulmonary fibrosis [69]. Moreover, MMPs expression is associated to GB invasion, growth and angiogenesis [70, 71]. MMP2 and MMP9 co-silencing in combination with temozolomide treatment emerge as promising in the treatment against GB [72]. Furthermore, phase II clinical trials using the broad-spectrum MMP-inhibitor, marimastat, in conjunction with temozolomide has shown encouraging results [73]. Thus, the evidences suggest a central role for WNT pathway and specific MMPs in GB and emerge as promising targets for potential treatments [43].

As a result, the tandem JNK-Wg-TMs trigger the expression of MMPs in Drosophila GB cells. Fly MMP1 and MMP2 degrade the ECM and, in the brain facilitate the infiltration of TMs. MMPs are upregulated in human patients [43] as well as in Drosophila GB model. Besides, MMPs expression attenuation reduces the volume occupied by $\mathrm{TMs}$ and $\mathrm{Wg}$ 
signalling with the concomitant consequences: reduction of GB progression, prevention of neurodegeneration (synapse loss) and lethality. Consequently, MMPs are part of the positive feedback loop in GB and mediate the equilibrium among Wg signalling, JNK and TMs expansion.

MMPs have been a field of interest for GB for more than a decade, numerous studies correlate MMPs expression with a poor prognosis $[43,74]$ but the precise mechanisms mediating the cellular impact of MMPs and the regulation of MMPs expression has not been elucidated. We propose that GB cells become addicted to signals independent of the founder mutations (PI3K and EGFR), in this case, the positive feedback loop formed by Wg pathway, JNK, MMPs and the TMs (Figure 9H). As a consequence, treatments tackling EGFR of PI3K failed to success as GB cells rely of other inputs. This particular signalling loop is required for GB cells to progress but is dispensable for normal glia development which puts these discoveries as targetable features for GB treatments.

Finally, the specific targets of MMPs will be of interest to determine which ECM components are essential to prevent TMs expansion. There are two MMPs in Drosophila (MMP1 and MMP2) with 10 and 3 isoforms respectively, MMP1 and MMP2 have been classically differentiated by their extracellular or membrane associated localization, however this concept is currently under debate [15]. It is proposed that each MMP has particular targets from the ECM which are sensible of degradation, recent classifications have brought light on the specificity of each protease and the different substrates [43]. The results show that the attenuation of either MMP1 or MMP2 prevents GB progression, thus the potential substrates and the functional implications for GB need to be established in future studies.

\section{Acknowledgements}


We thank Professor Alberto Ferrús and anonymous reviewers for critiques of the manuscript and for helpful discussions. We are grateful to R. Read, I. Guerrero, P. Leopold, C. Klambt, JP. Vincent, E. Martín-Blanco, K. Broadie, E. Moreno, the Vienna Drosophila Resource Centre, the Bloomington Drosophila stock Centre and the Developmental Studies Hydridoma Bank for supplying fly stocks and antibodies, and FlyBase for its wealth of information. We acknowledge the support of the Confocal Microscopy unit and Molecular Biology unit at the Cajal Institute for their help with this project. MP holds a fellowship from the Juan de la Cierva program IJCI-2014-19272 and SCT holds a contract from the Ramón y Cajal program RYC-2012-11410 from the Spanish MICINN. Research has been funded by grant BFU2015-65685P. Authors declare no conflicts of interest.

\section{Experimental Procedures}

\section{Fly stocks}

Flies were raised in standard fly food at $25^{\circ} \mathrm{C}$. Fly stocks from the Bloomington stock Centre: UAS-GFP ${ }^{n / s}$ (BL4776), UAS-lacZ (BL8529), UAS-myr-RFP (BL7119), UASGap43-RNAi (BL29598), repo-Gal4 (BL7415), UAS-CD8-GFP (BL32186), tub-gal80 ts (BL7019), Repo-lexA (a gift from C Klambt), lexAop-CD2-GFP (BL32205), UAS-bsk ${ }^{D N}$ (BL9311), egr-GFP (BL66381), egr ${ }^{[M / 15372]}$ (BL59754), en-Gal4, UAS-GFP (from BL25752), MMP2-GFP (BL60512). Fly stocks from the Vienna Drosophila Resource Centre: UAS-fz1-RNAi (v105493), UAS-mmp1-RNAi (v101505), UAS-mmp2-RNAi (v107888), lexAop-Fz1 [13], UAS-dEGFR', UAS-PI3K92E (dp110 CAAX) (A gift from R. Read), UAS-ihog-RFP (a gift from I. Guerrero), TRE-RFP-1b (a gift from J.P. Vincent)

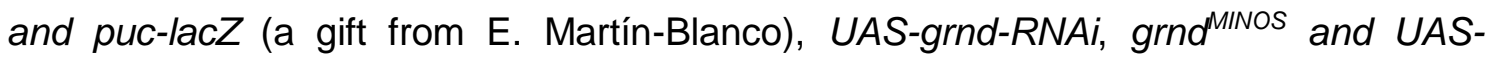
grnd $^{\text {EXTRA }}$ (a gift from P. Leopold) [75], UAS-dmyc (a gift from E. Moreno) [76], UAS-TOR$D E R^{C A}[77]$ 


\section{Drosophila glioblastoma model}

The most frequent genetic lesions in human gliomas include mutation or amplification of the Epidermal Growth Factor Receptor (EGFR) gene. Glioma-associated EGFR mutant forms show constitutive kinase activity that chronically stimulates Ras signaling to drive cell proliferation and migration $[78,79]$ Other common genetic lesions include loss of the lipid phosphatase PTEN, which antagonizes the phosphatidylinositol-3 kinase (PI3K) signaling pathway, and mutations that activate PI3KCA, which encodes the p110a catalytic subunit of PI3K ${ }^{98-99}$. Gliomas often show constitutively active Akt, a major PI3K effector. However, EGFR-Ras or PI3K mutations alone are not sufficient to transform glial cells. Instead, multiple mutations that coactivate EGFR-Ras and PI3K/Akt pathways are required to induce a glioma [80]. In Drosophila, a combination of EGFR and PI3K mutations effectively causes a glioma-like condition that shows features of human gliomas including glia expansion, brain invasion, neuron dysfunction, synapse loss and neurodegeneration [26, 81, 82]. Moreover, this model has proved to be useful in finding new kinase activities relevant to glioma progression [83] To generate a glioma in Drosophila melanogaster adult flies, the Gal4/UAS system [84] was used as described above (repo-Gal4>UAS-EGFRA,UAS-dp110. To restrict the expression of this genetic combination to the adulthood, we used the thermo sensitive repression system Gal $80^{\top \mathrm{TS}}$. Individuals maintained at $17^{\circ} \mathrm{C}$ did not activate the expression of the UAS constructs, but when flies were switched to $29^{\circ} \mathrm{C}$, the protein Gal80 $80^{\mathrm{TS}}$ changed conformation and was not longer able to bind to Gal4 to prevent its interaction with UAS sequences, and the expression system was activated. 


\section{Antibodies for Immunofluorescence}

Third-instar larval brains, were dissected in phosphate-buffered saline (PBS), fixed in 4\% formaldehyde for $30 \mathrm{~min}$, washed in PBS +0.1 or $0.3 \%$ Triton $\mathrm{X}-100$ (PBT), and blocked in $\mathrm{PBT}+5 \% \mathrm{BSA}$.

Antibodies used were: mouse anti-Wg (DSHB 1:50), mouse anti-Repo (DSHB 1:50), mouse anti-Fz1 (DSHB 1:50), mouse anti-Cyt-Arm (DSHB 1:50), mouse anti-MMP1 (DSHB 5H7B11, 3A6B4, 3B8D12, 1:50), rabbit anti-MMP2 (1:500, K. Broadie) [85],

guinea pig anti-grnd (1:250, P. Leopold) [75], mouse anti- $\beta$-galactosidase (Sigma, 1:500), rabbit anti-GFP (Invitrogen A11122, 1:500), mouse anti-GFP (Invitrogen A11120, 1:500), mouse anti-brp (DSHB Nc82, 1:20), Rabbit anti-Hrp (Jackson Immunoresearch 111-035-144, 1:400).

Secondary antibodies: anti-mouse Alexa 488, 568, 647, anti-rabbit Alexa 488, 568, 647 (Thermofisher, 1:500). DNA was stained with 2-(4-amidinophenyl)-1H-indole-6carboxamidine (DAPI, $1 \mu \mathrm{M})$.

\section{Western blots}

For western blots, we used NuPAGE Bis-Tris Gels 4-12\% (Invitrogen), and the following primary antibodies: mouse anti-MMP1 (DSHB 1:500) and mouse anti-tubulin (1:10,000 Sigma), we use Tubulin as a loading control instead of actin because the tumor microtubes are Actin positive and tubulin negative as previously described [10]. There were 3 biological replicates and Relative MMP1 Average pixel intensity was measured using measurement tool from Image Studio Lite Ver 5.2 and normalized against Tubulin.

\section{Survival assay}


Males Tub-Gal80; Repo-Gal4 were crossed with females bearing a control construct (UAS-LacZ) or glioma (UAS-PI3K ${ }^{\text {dp110 }}$ UAS-EGFR') or glioma + Mmp1-RNAi and glioma $+M m p 2-R N A i$ and raised at $17^{\circ} \mathrm{C}$. Progeny bearing a glioma or glioma $+\mathrm{Mmp} 1-$ RNAi and glioma + Mmp2-RNAi (experimental) or LacZ (control) chromosomes were put at $29^{\circ} \mathrm{C}$ and viability was calculated as the percentage of surviving flies with respect to the starting number of flies as follows: viability = observed ( $n^{\circ}$ of flies)/starting $n^{\circ}$ of flies $\times$ 100. Six independent vials for experimental $(n=6)$ and controls $(n=6)$ were analyzed, with each vial with 10 flies.

\section{Imaging}

Fluorescent labeled samples were mounted in Vectashield mounting media with DAPI (Vector Laboratories) and analyzed by Confocal microscopy (LEICA TCS SP5). Images were processed using Leica LAS AF Lite and Fiji (Image J 1.50e). Images were assembled using Adobe Photoshop CS5.1.

\section{Quantifications}

Relative Wg, Fz1, Cyt-Arm, TRE-RFP, grnd, MMP1 and MMP2 staining within brains was determined from images taken at the same confocal settings. Average pixel intensity was measured using measurement log tool from Fiji 1.51g and Adobe Photoshop CS5.1. Average pixel intensity was measured in the glial tissue and in the adjacent neuronal tissue ( $\mathrm{N}<10$ for each sample in triplicates) and expressed as a Glia/Neuron ratio in all cases but Cyt-Arm that was expressed as Neuron/Glia ratio. Glial network volume was quantified using Imaris surface tool (Imaris 6.3.1 software).

For the co-localization of egr-GFP in glial cells, GFP channel volume was quantified using Imaris surface tool. We selected a specific threshold for the total volume in the 
control samples and then we applied these conditions to the analysis of the corresponding experimental sample. Then we applied a co-localization filter (intensity center of the red channel)

The number of Repo ${ }^{+}$cells, the number of synaptic active sites and the number of puclacZ positive cells was quantified by using the spots tool Imaris 6.3 .1 software, we selected a minimum size and threshold for the puncta in the control samples of each experiment. Then we applied these conditions to the analysis of each corresponding experimental sample. For the puc-lacZ glia or neuron co-localization studies we quantified the total number of puc-lac $Z^{+}$cells and then applied a co-localization filter (intensity center of the channel of interest) using the Spots tool from the Imaris 6.3.1 Bitplane Scientific Solutions software.

\section{Statistical Analysis}

To analyze and plot the data, we used Microsoft Excel 2013 and GraphPad Prism 6. We performed a D'Agostino \& Pearson normality test and the data found to have a normal distribution were analyzed by a two-tailed t test with Welch-correction. In the case of multiple comparisons, we used a One-way ANOVA with Bonferroni post-test. The data that did not pass the normality test were subjected to a two-tailed Mann-Whitney $U$ test or in the case of multiple comparisons a Kruskal-Wallis test with Dunns post-test. Error bars represent standard error of the mean. * represents $p$ value $\leq .05 ;{ }^{* *} p$ value $\leq .01$; ${ }^{* * *} p$ value $\leq .001$. Statistical values of $p$ value $>.05$ were not considered significant, (n.s.). 


\section{Figure 1: MMP1 is upregulated in GB}

Brains from 3rd instar larvae displayed at the same scale. Glia are labeled with UASIhog-RFP driven by repo-Gal4 to visualize active cytonemes/ TM structures in glial cells, and stained with MMP1 (green). (A) MMP1 is homogeneously distributed in control sections, with a slight accumulation in the lhog+ projections (B) MMP1 accumulates in the TMs and specifically in the projections that are in contact with the neuronal clusters. (C) Inhibition of Gap43 by RNAi in glioma brains restores a normal glial network and MMP1 does not accumulate showing a homogeneous staining along the brain section. (D) Inhibition of $F z 1$ by $R N A i$ in glioma brains restores a normal MMP1 distribution. MMP1 does not accumulate showing a homogeneous staining along the brain section. Nuclei are marked with DAPI. (E) Quantification of MMP1 staining ratio between ihog ${ }^{+}$ and ihog- domains. (F) Western blot of samples extracted from control, glioma, glioma Gap43-RNAi and Glioma Fz1-RNAi larvae showing changes in the amount of MMP1 or protein. Error bars show S.D. * $\mathrm{P}<0.01$, ${ }^{* *} \mathrm{P}<0.001$, ${ }^{* \star *} \mathrm{P}<0.0001$ or ns for nonsignificant. Scale bar size is indicated in this and all figures.

Figure 2: Wg pathway is necessary and sufficient to activate MMP expression in glial cells

Brains from 3rd instar larvae displayed at the same scale. Glial cell bodies and membranes labelled in green (CD8-GFP) driven by repo-Gal4 to the glial cells and stained with MMP1 (red). (A-B) MMP1 is homogeneously distributed in control sections. (C-D) MMP1 accumulates in the glial cells upon Fz1 overexpression. (E) Quantification of MMP1 staining ratio between $\mathrm{GFP}^{+}$and $\mathrm{GFP}^{-}$domains. Nuclei are marked with DAPI. Error bars show S.D. ${ }^{*} \mathrm{P}<0.01,{ }^{* *} \mathrm{P}<0.001,{ }^{* \star *} \mathrm{P}<0.0001$ or ns for non-significant. Scale bar size is indicated in this and all figures.

Figure 3: JNK signaling pathway activation in glioma 
Larval brain sections from 3rd instar larvae displayed at the same scale. Glial cell bodies and membranes are labeled with UAS-myr-RFP (red) driven by repo-Gal4 (A-C) JNK signaling pathway reporter puc-lacZ in control, glioma 48h and glioma 96h After Tumour induction, shows activation of the pathway mostly in neurons in control samples (A) and then shows a progressive activation in glial transformed cells (B-C). (D) Quantification of the $\%$ of cells with Puc-lacZ activation in glial cells and neurons domains.

Glial cell nuclei are labeled with UAS-GFP ${ }^{N L S}$ (Green) driven by repo-Gal4. (E-H) JNK signaling pathway reporter TRE-RFP in control, glioma, glioma Gap43-RNAi and Glioma Fz1-RNAi brain sections. The number of glial cells is quantified in (I) and the TRE-RFP average pixel intensity per glial cells is quantified in (J). Nuclei are marked with DAPI. Error bars show S.D. ${ }^{*} \mathrm{P}<0.01,{ }^{* *} \mathrm{P}<0.001,{ }^{* *} \mathrm{P}<0.0001$ or $\mathrm{ns}$ for non-significant. Scale bar size is indicated in this and all figures.

\section{Figure 4: TNF receptor Grindelwald mediates GB progression}

Brains from 3rd instar larvae displayed at the same scale. Glia are labeled with UASIhog-RFP driven by repo-Gal4 to visualize active cytonemes/ TM structures in glial cells, and stained with Repo (green) in the following genotypes (A-F) control, glioma, grndRNAi, glioma grnd-RNAi, grnd ${ }^{-/-}$and Glioma grnd $^{-/-}$brain sections. The number of Repo ${ }^{+}$ cells is quantified in (G). Nuclei are marked with DAPI. Error bars show S.D. * $P<0.01$, ${ }^{* *} \mathrm{P}<0.001,{ }^{* *} \mathrm{P}<0.0001$ or ns for non-significant. Scale bar size is indicated in this and all figures.

Figure 5: JNK pathway is necessary to localize Fz1 in the TMs and promote GB progression

Brains from 3rd instar larvae displayed at the same scale. Glia are labeled with UASIhog-RFP driven by repo-Gal4 to visualize active cytonemes/ TM structures in glial cells, and stained with Fz1 (green) in the following genotypes (A-F) control, glioma, grnd-RNAi, glioma grnd-RNAi, grnd $^{-/-}$and Glioma grnd $^{-/-}$brain sections. Fz1 average pixel intensity 
staining quantification ratio between ihog ${ }^{+}$and ihog ${ }^{-}$domains $(G)$. Nuclei are marked with DAPI. Error bars show S.D. * $\mathrm{P}<0.01$, ${ }^{* *} \mathrm{P}<0.001$, ${ }^{* *} \mathrm{P}<0.0001$ or ns for nonsignificant. Scale bar size are indicated in this and all figures.

\section{Figure 6: grnd mediates Wg pathway activation in GB}

Brains from 3rd instar larvae displayed at the same scale. Glia are labeled with UASIhog-RFP driven by repo-Gal4 to visualize active cytonemes/ TM structures in glial cells, and stained with Cyt-Arm (green) in the following genotypes (A-F) control, glioma, grndRNAi, glioma grnd-RNAi, grnd ${ }^{-/-}$and Glioma grnd $^{-/-}$brain sections. (A) CytoplasmicArmadillo (Cyt-Arm) is homogeneously distributed in control sections. (B) In glioma brains Cyt-Arm accumulates in the neurons cytoplasm where it is inactive. (C-F) Knockdown or knockout of grnd in both normal or glioma brains shows a normal glial network and Cyt-Arm does not accumulate showing a homogeneous distribution similar to the control. Cyt-Arm average pixel intensity quantification for Neuron/Glia ratio between ihog and ihog ${ }^{+}$domains $(G)$. Nuclei are marked with DAPI. Error bars show S.D. ${ }^{*} \mathrm{P}<0.01,{ }^{* *} \mathrm{P}<0.001,{ }^{* * *} \mathrm{P}<0.0001$ or $\mathrm{ns}$ for non-significant. Scale bar size is indicated in this and all figures.

\section{Figure 7: JNK triggers MMPs expression in GB}

Brains from 3rd instar larvae displayed at the same scale. Glia is labelled with UAS-IhogRFP driven by repo-Gal4 to visualize active cytonemes/ TM structures in glial cells, and stained with MMP1 (green). (A) MMP1 is homogeneously distributed in control sections (B) MMP1 accumulates in the TMs and specifically in the projections that are in contact with the neuronal clusters. (C-E) Blocking JNK pathway by using a UAS-bsk ${ }^{D N}$ or UASgrnd-RNAi in glioma brains restores a normal glial network and MMP1 does not accumulate showing a homogeneous staining along the brain section. (F) Quantification of MMP1 staining ratio between ihog ${ }^{+}$and ihog $^{-}$domains. Error bars show S.D. ${ }^{*} P<0.01$, 
${ }^{* *} \mathrm{P}<0.001,{ }^{* * *} \mathrm{P}<0.0001$ or ns for non-significant. Scale bar size are indicated in this and all figures.

\section{Figure 8: TMs expansion requires MMPs}

Brains from 3rd instar larvae displayed at the same scale. Glia are labeled with UASIhog-RFP driven by repo-Gal4 to visualize active cytonemes/ TM structures in glial cells, and stained with Fz1 (green) in the following genotypes (A-F) control, glioma, MMP1RNAi, glioma MMP1-RNAi, MMP2-RNAi and glioma MMP2-RNAi brain sections. (G) Quantification of Fz1 average pixel intensity staining ratio between ihog ${ }^{+}$and ihog $^{-}$ domains. (H) Quantification of glial/glioma network volume expansion. Nuclei are marked with DAPI. (I) Survival curve of adult control, glioma, glioma MMP1-RNAi and glioma MMP2-RNAi flies after a number of days of glioma induction and progression. Error bars show S.D. ${ }^{*} \mathrm{P}<0.01,{ }^{* *} \mathrm{P}<0.001,{ }^{* * *} \mathrm{P}<0.0001$ or ns for non-significant. Scale bar size is indicated in this and all figures.

Figure 9: MMPs upregulation in glioma are responsible for the synapse loss in the neurons

Neurons from the larval neuromuscular junction are stained with Nc82 (brp) showing the synaptic active Zones in grey. (A-F) Upon glioma induction (B) the number of synapses (grey) is reduced when compared with the control (A). The number of synapses is restored upon knockdown of MMP1 or MMP2 $(\mathrm{D}, \mathrm{F})$. The quantification of the number of synaptic active zones in all genotypes is shown in $(\mathrm{G}) .(\mathrm{H})$ Model: Glial cells are initially transformed into malignant GB upon EGFR and PI3K pathways constitutive activation, afterwards GB cells establish a positive feedback loop including TMs, Wg signalling, JNK and matrix metalloproteinases (MMPs). Initial stimulation of actin cytoskeleton remodelling via EGFR/PI3K enables initial expansion of TMs, as a consequence Frizzled1 receptor accumulation in TMs mediates neuronal $\mathrm{Wg}$ depletion and $\mathrm{Wg}$ 
signalling upregulation in the GB cells which activates JNK in GB. As a consequence, MMPs are upregulated and facilitate further TMs infiltration in the brain, hence GB TMs network expands and mediate further wingless depletion to close the loop.

1. Gallego O. Nonsurgical treatment of recurrent glioblastoma. Curr Oncol. 2015;22(4):e273-81. Epub 2015/08/25. doi: 10.3747/co.22.2436

conc-22-e273 [pii]. PubMed PMID: 26300678; PubMed Central PMCID: PMC4530825. 2. $\quad$ McGuire S. World Cancer Report 2014. Geneva, Switzerland: World Health Organization, International Agency for Research on Cancer, WHO Press, 2015. Adv Nutr. 2016;7(2):418-9. Epub 2016/03/17. doi: 7/2/418 [pii]

10.3945/an.116.012211. PubMed PMID: 26980827; PubMed Central PMCID: PMC4785485. 3. Rogers TW, Toor G, Drummond K, Love C, Field K, Asher R, et al. The 2016 revision of the WHO Classification of Central Nervous System Tumours: retrospective application to a cohort of diffuse gliomas. J Neurooncol. 2018;137(1):181-9. Epub 2017/12/09. doi: 10.1007/s11060-017-2710-7

10.1007/s11060-017-2710-7 [pii]. PubMed PMID: 29218432.

4. Louis DN, Perry A, Reifenberger G, von Deimling A, Figarella-Branger D, Cavenee WK, et al. The 2016 World Health Organization Classification of Tumors of the Central Nervous System: a summary. Acta Neuropathol. 2016;131(6):803-20. Epub 2016/05/10. doi: 10.1007/s00401-016-1545-1

10.1007/s00401-016-1545-1 [pii]. PubMed PMID: 27157931.

5. Alifieris C, Trafalis DT. Glioblastoma multiforme: Pathogenesis and treatment. Pharmacol Ther. 2015;152:63-82. Epub 2015/05/07. doi: S0163-7258(15)00096-0 [pii]

10.1016/j.pharmthera.2015.05.005. PubMed PMID: 25944528.

6. Hayden EC. Genomics boosts brain-cancer work. Nature. 2010;463(7279):278. Epub 2010/01/22. doi: 463278a [pii]

10.1038/463278a. PubMed PMID: 20090720.

7. Wick W, Osswald M, Wick A, Winkler F. Treatment of glioblastoma in adults. Ther Adv Neurol Disord. 2018;11:1756286418790452. Epub 2018/08/08. doi: $10.1177 / 1756286418790452$

10.1177_1756286418790452 [pii]. PubMed PMID: 30083233; PubMed Central PMCID: PMC6071154.

8. Waitkus MS, Diplas BH, Yan H. Biological Role and Therapeutic Potential of IDH Mutations in Cancer. Cancer Cell. 2018;34(2):186-95. Epub 2018/05/29. doi: S15356108(18)30182-X [pii] 
9. Miller JJ, Shih HA, Andronesi OC, Cahill DP. Isocitrate dehydrogenase-mutant glioma: Evolving clinical and therapeutic implications. Cancer. 2017;123(23):4535-46. Epub 2017/10/06. doi: 10.1002/cncr.31039. PubMed PMID: 28980701.

10. Osswald M, Jung E, Sahm F, Solecki G, Venkataramani V, Blaes J, et al. Brain tumour cells interconnect to a functional and resistant network. Nature. 2015;528(7580):93-8. Epub 2015/11/05. doi: nature16071 [pii]

10.1038/nature16071. PubMed PMID: 26536111.

11. Osswald M, Solecki G, Wick W, Winkler F. A malignant cellular network in gliomas: potential clinical implications. Neuro Oncol. 2016;18(4):479-85. Epub 2016/03/21. doi: now014 [pii]

10.1093/neuonc/now014. PubMed PMID: 26995789; PubMed Central PMCID: PMC4799690. 12. Arnes M, Casas Tinto S. Aberrant Wnt signaling: a special focus in CNS diseases. J Neurogenet. 2017;31(4):216-22. Epub 2017/06/22. doi: 10.1080/01677063.2017.1338696. PubMed PMID: 28635355.

13. Portela M. VV, Fahey-Lozano N., Seco E., Losada-Perez M., Winkler F, Casas-Tinto S. Active WNT vampirization by glioblastoma network leads to brain tumor growth and neurodegeneration. BioRxiv. 2018;428953. doi: https://doi.org/10.1101/428953.

14. Malemud CJ. Matrix metalloproteinases (MMPs) in health and disease: an overview. Front Biosci. 2006;11:1696-701. Epub 2005/12/22. doi: 1915 [pii]. PubMed PMID: 16368548. 15. LaFever KS, Wang X, Page-McCaw P, Bhave G, Page-McCaw A. Both Drosophila matrix metalloproteinases have released and membrane-tethered forms but have different substrates. Sci Rep. 2017;7:44560. Epub 2017/03/17. doi: srep44560 [pii]

10.1038/srep44560. PubMed PMID: 28300207; PubMed Central PMCID: PMC5353688. 16. Veeravalli KK, Rao JS. MMP-9 and UPAR regulated glioma cell migration. Cell Adh Migr. 2012;6(6):509-12. Epub 2012/10/19. doi: 21673 [pii]

10.4161/cam.21673. PubMed PMID: 23076139; PubMed Central PMCID: PMC3547895. 17. de Lucas AG, Schuhmacher AJ, Oteo M, Romero E, Camara JA, de Martino A, et al. Targeting MT1-MMP as an ImmunoPET-Based Strategy for Imaging Gliomas. PLoS One. 2016;11(7):e0158634. Epub 2016/07/28. doi: 10.1371/journal.pone.0158634

PONE-D-15-48101 [pii]. PubMed PMID: 27462980; PubMed Central PMCID: PMC4962974. 18. Nakada M, Okada Y, Yamashita J. The role of matrix metalloproteinases in glioma invasion. Front Biosci. 2003;8:e261-9. Epub 2002/11/29. PubMed PMID: 12456313.

19. Yamamoto $M$, Ueno $Y$, Hayashi $S$, Fukushima $T$. The role of proteolysis in tumor invasiveness in glioblastoma and metastatic brain tumors. Anticancer Res. 2002;22(6C):4265-8. Epub 2003/01/30. PubMed PMID: 12553067.

20. Matsuda K, Sato A, Okada M, Shibuya K, Seino S, Suzuki K, et al. Targeting JNK for therapeutic depletion of stem-like glioblastoma cells. Sci Rep. 2012;2:516. Epub 2012/07/21. doi: 10.1038/srep00516. PubMed PMID: 22816039; PubMed Central PMCID: PMC3400080.

21. Zeigler ME, Chi Y, Schmidt T, Varani J. Role of ERK and JNK pathways in regulating cell motility and matrix metalloproteinase 9 production in growth factor-stimulated human epidermal keratinocytes. J Cell Physiol. 1999;180(2):271-84. Epub 1999/07/08. doi: 10.1002/(SICI)1097-4652(199908)180:2<271::AID-JCP15>3.0.CO;2-D [pii]

10.1002/(SICI)1097-4652(199908)180:2<271::AID-JCP15>3.0.CO;2-D. PubMed PMID: 10395297.

22. Lee YS, Lan Tran HT, Van Ta Q. Regulation of expression of matrix metalloproteinase-9 by JNK in Raw 264.7 cells: presence of inhibitory factor(s) suppressing MMP-9 induction in serum and conditioned media. Exp Mol Med. 2009;41(4):259-68. Epub 2009/03/21. doi: 
10.3858/emm.2009.41.4.029. PubMed PMID: 19299915; PubMed Central PMCID: PMC2679232.

23. Uhlirova M, Bohmann D. JNK- and Fos-regulated Mmp1 expression cooperates with Ras to induce invasive tumors in Drosophila. EMBO J. 2006;25(22):5294-304. Epub 2006/11/04. doi: 7601401 [pii]

10.1038/sj.emboj.7601401. PubMed PMID: 17082773; PubMed Central PMCID: PMC1636619.

24. Cheng CY, Hsieh HL, Hsiao LD, Yang CM. PI3-K/Akt/JNK/NF-kappaB is essential for MMP-9 expression and outgrowth in human limbal epithelial cells on intact amniotic membrane. Stem Cell Res. 2012;9(1):9-23. Epub 2012/03/31. doi: S1873-5061(12)00024-4 [pii]

10.1016/j.scr.2012.02.005. PubMed PMID: 22459175.

25. Ispanovic E, Haas TL. JNK and PI3K differentially regulate MMP-2 and MT1-MMP mRNA and protein in response to actin cytoskeleton reorganization in endothelial cells. Am J Physiol Cell Physiol. 2006;291(4):C579-88. Epub 2006/05/05. doi: 00300.2005 [pii]

10.1152/ajpcell.00300.2005. PubMed PMID: 16672691.

26. Read RD, Cavenee WK, Furnari FB, Thomas JB. A drosophila model for EGFR-Ras and PI3K-dependent human glioma. PLoS Genet. 2009;5(2):e1000374. Epub 2009/02/14. doi: 10.1371/journal.pgen.1000374. PubMed PMID: 19214224; PubMed Central PMCID: PMCPMC2636203.

27. Winkler F, Wick W. Harmful networks in the brain and beyond. Science. 2018;359(6380):1100-1. Epub 2018/03/29. doi: 359/6380/1100 [pii]

10.1126/science.aar5555. PubMed PMID: 29590028.

28. Bischoff M, Gradilla AC, Seijo I, Andres G, Rodriguez-Navas C, Gonzalez-Mendez L, et al. Cytonemes are required for the establishment of a normal Hedgehog morphogen gradient in Drosophila epithelia. Nat Cell Biol. 2013;15(11):1269-81. Epub 2013/10/15. doi: 10.1038/ncb2856. PubMed PMID: 24121526; PubMed Central PMCID: PMCPMC3840581. 29. Huang C, Rajfur Z, Borchers C, Schaller MD, Jacobson K. JNK phosphorylates paxillin and regulates cell migration. Nature. 2003;424(6945):219-23. Epub 2003/07/11. doi: 10.1038/nature01745

nature01745 [pii]. PubMed PMID: 12853963.

30. Hagemann T, Wilson J, Kulbe H, Li NF, Leinster DA, Charles K, et al. Macrophages induce invasiveness of epithelial cancer cells via NF-kappa B and JNK. J Immunol. 2005;175(2):1197-205. Epub 2005/07/09. doi: 175/2/1197 [pii]. PubMed PMID: 16002723. 31. Zeng A, Yin J, Li Y, Li R, Wang Z, Zhou X, et al. miR-129-5p targets Wnt5a to block PKC/ERK/NF-kappaB and JNK pathways in glioblastoma. Cell Death Dis. 2018;9(3):394. Epub 2018/03/14. doi: 10.1038/s41419-018-0343-1

10.1038/s41419-018-0343-1 [pii]. PubMed PMID: 29531296; PubMed Central PMCID: PMC5847604.

32. Mu N, Gu J, Liu N, Xue X, Shu Z, Zhang K, et al. PRL-3 is a potential glioblastoma prognostic marker and promotes glioblastoma progression by enhancing MMP7 through the ERK and JNK pathways. Theranostics. 2018;8(6):1527-39. Epub 2018/03/21. doi: 10.7150/thno.22699

thnov08p1527 [pii]. PubMed PMID: 29556339; PubMed Central PMCID: PMC5858165. 33. Kitanaka C, Sato A, Okada M. JNK Signaling in the Control of the Tumor-Initiating Capacity Associated with Cancer Stem Cells. Genes Cancer. 2013;4(9-10):388-96. Epub 2013/12/19. doi: 10.1177/1947601912474892 
10.1177_1947601912474892 [pii]. PubMed PMID: 24349636; PubMed Central PMCID: PMC3863334.

34. Feng J, Yan PF, Zhao HY, Zhang FC, Zhao WH, Feng M. Inhibitor of Nicotinamide Phosphoribosyltransferase Sensitizes Glioblastoma Cells to Temozolomide via Activating ROS/JNK Signaling Pathway. Biomed Res Int. 2016;2016:1450843. Epub 2017/01/18. doi: 10.1155/2016/1450843. PubMed PMID: 28097126; PubMed Central PMCID: PMC5206411. 35. Okada M, Sato A, Shibuya K, Watanabe E, Seino S, Suzuki S, et al. JNK contributes to temozolomide resistance of stem-like glioblastoma cells via regulation of MGMT expression. Int J Oncol. 2014;44(2):591-9. Epub 2013/12/10. doi: 10.3892/ijo.2013.2209. PubMed PMID: 24316756.

36. Martin-Blanco E, Gampel A, Ring J, Virdee K, Kirov N, Tolkovsky AM, et al. puckered encodes a phosphatase that mediates a feedback loop regulating JNK activity during dorsal closure in Drosophila. Genes Dev. 1998;12(4):557-70. Epub 1998/03/21. PubMed PMID: 9472024; PubMed Central PMCID: PMCPMC316530.

37. Langen M, Koch M, Yan J, De Geest N, Erfurth ML, Pfeiffer BD, et al. Mutual inhibition among postmitotic neurons regulates robustness of brain wiring in Drosophila. Elife. 2013;2:e00337. Epub 2013/03/09. doi: 10.7554/eLife.00337. PubMed PMID: 23471010; PubMed Central PMCID: PMCPMC3589824.

38. Chatterjee N, Bohmann D. A versatile PhiC31 based reporter system for measuring AP1 and Nrf2 signaling in Drosophila and in tissue culture. PLoS One. 2012;7(4):e34063. Epub 2012/04/18. doi: 10.1371/journal.pone.0034063. PubMed PMID: 22509270; PubMed Central PMCID: PMCPMC3324472.

39. Jemc JC, Milutinovich AB, Weyers JJ, Takeda Y, Van Doren M. raw Functions through JNK signaling and cadherin-based adhesion to regulate Drosophila gonad morphogenesis. Dev Biol. 2012;367(2):114-25. Epub 2012/05/12. doi: 10.1016/j.ydbio.2012.04.027. PubMed PMID: 22575490; PubMed Central PMCID: PMCPMC3635074.

40. Ruan W, Srinivasan A, Lin S, Kara k I, Barker PA. Eiger-induced cell death relies on Rac1dependent endocytosis. Cell Death Dis. 2016;7:e2181. Epub 2016/04/08. doi: cddis201680 [pii]

10.1038/cddis.2016.80. PubMed PMID: 27054336; PubMed Central PMCID: PMC4855659.

41. Weber U, Paricio N, Mlodzik M. Jun mediates Frizzled-induced R3/R4 cell fate distinction and planar polarity determination in the Drosophila eye. Development.

2000;127(16):3619-29. Epub 2000/07/21. PubMed PMID: 10903185.

42. Riggleman B, Schedl P, Wieschaus E. Spatial expression of the Drosophila segment polarity gene armadillo is posttranscriptionally regulated by wingless. Cell. 1990;63(3):549-60. Epub 1990/11/02. doi: 0092-8674(90)90451-J [pii]. PubMed PMID: 2225066.

43. Hagemann C, Anacker J, Ernestus RI, Vince GH. A complete compilation of matrix metalloproteinase expression in human malignant gliomas. World J Clin Oncol. 2012;3(5):6779. Epub 2012/05/15. doi: 10.5306/wjco.v3.i5.67. PubMed PMID: 22582165; PubMed Central PMCID: PMC3349915.

44. Hagemann C, Anacker J, Haas S, Riesner D, Schomig B, Ernestus RI, et al. Comparative expression pattern of Matrix-Metalloproteinases in human glioblastoma cell-lines and primary cultures. BMC Res Notes. 2010;3:293. Epub 2010/11/12. doi: 1756-0500-3-293 [pii]

10.1186/1756-0500-3-293. PubMed PMID: 21067565; PubMed Central PMCID: PMC2996401. 45. Munaut C, Noel A, Hougrand O, Foidart JM, Boniver J, Deprez M. Vascular endothelial growth factor expression correlates with matrix metalloproteinases MT1-MMP, MMP-2 and MMP-9 in human glioblastomas. Int J Cancer. 2003;106(6):848-55. Epub 2003/08/15. doi: 10.1002/ijc.11313. PubMed PMID: 12918061.

46. Lyu J, Joo CK. Wnt-7a up-regulates matrix metalloproteinase-12 expression and promotes cell proliferation in corneal epithelial cells during wound healing. J Biol Chem. 2005;280(22):21653-60. Epub 2005/04/02. doi: M500374200 [pii] 
10.1074/jbc.M500374200. PubMed PMID: 15802269.

47. Uraguchi M, Morikawa M, Shirakawa M, Sanada K, Imai K. Activation of WNT family expression and signaling in squamous cell carcinomas of the oral cavity. J Dent Res.

2004;83(4):327-32. Epub 2004/03/27. doi: 10.1177/154405910408300411. PubMed PMID: 15044508.

48. Lowy AM, Clements WM, Bishop J, Kong L, Bonney T, Sisco K, et al. beta-Catenin/Wnt signaling regulates expression of the membrane type 3 matrix metalloproteinase in gastric cancer. Cancer Res. 2006;66(9):4734-41. Epub 2006/05/03. doi: 66/9/4734 [pii]

10.1158/0008-5472.CAN-05-4268. PubMed PMID: 16651426.

49. Roomi MW, Kalinovsky T, Rath M, Niedzwiecki A. Modulation of MMP-2 and MMP-9 secretion by cytokines, inducers and inhibitors in human glioblastoma T-98G cells. Oncol Rep. 2017;37(3):1907-13. Epub 2017/01/24. doi: 10.3892/or.2017.5391. PubMed PMID: 28112361. 50. Page-McCaw A, Serano J, Sante JM, Rubin GM. Drosophila matrix metalloproteinases are required for tissue remodeling, but not embryonic development. Dev Cell. 2003;4(1):95106. Epub 2003/01/18. doi: S1534-5807(02)00400-8 [pii]. PubMed PMID: 12530966.

51. Kamino $M$, Kishida $M$, Kibe $T$, Ikoma $K$, lijima $M$, Hirano $H$, et al. Wnt-5a signaling is correlated with infiltrative activity in human glioma by inducing cellular migration and MMP-2. Cancer Sci. 2011;102(3):540-8. Epub 2011/01/06. doi: 10.1111/j.1349-7006.2010.01815.x. PubMed PMID: 21205070.

52. Roth W, Wild-Bode C, Platten M, Grimmel C, Melkonyan HS, Dichgans J, et al. Secreted Frizzled-related proteins inhibit motility and promote growth of human malignant glioma cells. Oncogene. 2000;19(37):4210-20. Epub 2000/09/12. doi: 10.1038/sj.onc.1203783. PubMed PMID: 10980594.

53. Chen W, Zhong X, Wei Y, Liu Y, Yi Q, Zhang G, et al. TGF-beta Regulates Survivin to Affect Cell Cycle and the Expression of EGFR and MMP9 in Glioblastoma. Mol Neurobiol. 2016;53(3):1648-53. Epub 2015/02/17. doi: 10.1007/s12035-015-9121-6

10.1007/s12035-015-9121-6 [pii]. PubMed PMID: 25682969.

54. Depetris-Chauvin A, Fernandez-Gamba A, Gorostiza EA, Herrero A, Castano EM, Ceriani MF. Mmp1 processing of the PDF neuropeptide regulates circadian structural plasticity of pacemaker neurons. PLoS Genet. 2014;10(10):e1004700. Epub 2014/10/31. doi: 10.1371/journal.pgen.1004700

PGENETICS-D-14-00171 [pii]. PubMed PMID: 25356918; PubMed Central PMCID: PMC4214601. 55. Bi WL, Beroukhim R. Beating the odds: extreme long-term survival with glioblastoma. Neuro Oncol. 2014;16(9):1159-60. Epub 2014/08/07. doi: nou166 [pii]

10.1093/neuonc/nou166. PubMed PMID: 25096192; PubMed Central PMCID: PMC4136904. 56. Arrazola MS, Silva-Alvarez C, Inestrosa NC. How the Wnt signaling pathway protects from neurodegeneration: the mitochondrial scenario. Front Cell Neurosci. 2015;9:166. Epub 2015/05/23. doi: 10.3389/fncel.2015.00166. PubMed PMID: 25999816; PubMed Central PMCID: PMC4419851.

57. Garcia-Velazquez $\mathrm{L}$, Arias $\mathrm{C}$. The emerging role of Wnt signaling dysregulation in the understanding and modification of age-associated diseases. Ageing Res Rev. 2017;37:135-45. Epub 2017/06/19. doi: S1568-1637(17)30090-9 [pii]

10.1016/j.arr.2017.06.001. PubMed PMID: 28624530.

58. Kahn M. Can we safely target the WNT pathway? Nat Rev Drug Discov. 2014;13(7):51332. Epub 2014/07/02. doi: nrd4233 [pii]

10.1038/nrd4233. PubMed PMID: 24981364; PubMed Central PMCID: PMC4426976. 
59. Libro R, Bramanti P, Mazzon E. The role of the Wnt canonical signaling in neurodegenerative diseases. Life Sci. 2016;158:78-88. Epub 2016/07/03. doi: S00243205(16)30380-0 [pii]

10.1016/j.Ifs.2016.06.024. PubMed PMID: 27370940.

60. Taylor TE, Furnari FB, Cavenee WK. Targeting EGFR for treatment of glioblastoma: molecular basis to overcome resistance. Curr Cancer Drug Targets. 2012;12(3):197-209. Epub 2012/01/25. doi: CCDT-EPUB-20120116-001 [pii]. PubMed PMID: 22268382; PubMed Central PMCID: PMC3464093.

61. Westphal M, Maire CL, Lamszus K. EGFR as a Target for Glioblastoma Treatment: An Unfulfilled Promise. CNS Drugs. 2017;31(9):723-35. Epub 2017/08/10. doi: 10.1007/s40263017-0456-6

10.1007/s40263-017-0456-6 [pii]. PubMed PMID: 28791656; PubMed Central PMCID: PMC5573763.

62. Prasad G, Sottero T, Yang X, Mueller S, James CD, Weiss WA, et al. Inhibition of $\mathrm{PI} 3 \mathrm{~K} / \mathrm{mTOR}$ pathways in glioblastoma and implications for combination therapy with temozolomide. Neuro Oncol. 2011;13(4):384-92. Epub 2011/02/15. doi: noq193 [pii]

10.1093/neuonc/noq193. PubMed PMID: 21317208; PubMed Central PMCID: PMC3064692. 63. Westhoff MA, Karpel-Massler G, Bruhl O, Enzenmuller S, La Ferla-Bruhl K, Siegelin MD, et al. A critical evaluation of PI3K inhibition in Glioblastoma and Neuroblastoma therapy. Mol Cell Ther. 2014;2:32. Epub 2014/01/01. doi: 10.1186/2052-8426-2-32

37 [pii]. PubMed PMID: 26056598; PubMed Central PMCID: PMC4452069.

64. Zhao HF, Wang J, Shao W, Wu CP, Chen ZP, To ST, et al. Recent advances in the use of PI3K inhibitors for glioblastoma multiforme: current preclinical and clinical development. Mol Cancer. 2017;16(1):100. Epub 2017/06/09. doi: 10.1186/s12943-017-0670-3

10.1186/s12943-017-0670-3 [pii]. PubMed PMID: 28592260; PubMed Central PMCID: PMC5463420.

65. McGranahan N, Swanton C. Clonal Heterogeneity and Tumor Evolution: Past, Present, and the Future. Cell. 2017;168(4):613-28. Epub 2017/02/12. doi: 10.1016/j.cell.2017.01.018. PubMed PMID: 28187284.

66. Qazi MA, Vora P, Venugopal C, Sidhu SS, Moffat J, Swanton C, et al. Intratumoral heterogeneity: pathways to treatment resistance and relapse in human glioblastoma. Ann Oncol. 2017;28(7):1448-56. Epub 2017/04/14. doi: 10.1093/annonc/mdx169. PubMed PMID: 28407030.

67. Wu B, Crampton SP, Hughes CC. Wnt signaling induces matrix metalloproteinase expression and regulates T cell transmigration. Immunity. 2007;26(2):227-39. Epub 2007/02/20. doi: 10.1016/j.immuni.2006.12.007. PubMed PMID: 17306568; PubMed Central PMCID: PMCPMC1855210.

68. Ingraham CA, Park GC, Makarenkova HP, Crossin KL. Matrix metalloproteinase (MMP)9 induced by Wnt signaling increases the proliferation and migration of embryonic neural stem cells at low O2 levels. J Biol Chem. 2011;286(20):17649-57. Epub 2011/04/05. doi: 10.1074/jbc.M111.229427. PubMed PMID: 21460212; PubMed Central PMCID: PMCPMC3093840.

69. Konigshoff M, Balsara N, Pfaff EM, Kramer M, Chrobak I, Seeger W, et al. Functional Wnt signaling is increased in idiopathic pulmonary fibrosis. PLoS One. 2008;3(5):e2142. Epub 2008/05/15. doi: 10.1371/journal.pone.0002142. PubMed PMID: 18478089; PubMed Central PMCID: PMCPMC2374879.

70. Lakka SS, Gondi CS, Yanamandra N, Olivero WC, Dinh DH, Gujrati M, et al. Inhibition of cathepsin B and MMP-9 gene expression in glioblastoma cell line via RNA interference reduces 
tumor cell invasion, tumor growth and angiogenesis. Oncogene. 2004;23(27):4681-9. Epub 2004/05/04. doi: 10.1038/sj.onc.1207616. PubMed PMID: 15122332.

71. Rome C, Arsaut J, Taris C, Couillaud F, Loiseau H. MMP-7 (matrilysin) expression in human brain tumors. Mol Carcinog. 2007;46(6):446-52. Epub 2007/01/16. doi:

10.1002/mc.20293. PubMed PMID: 17219436.

72. Gabelloni P, Da Pozzo E, Bendinelli S, Costa B, Nuti E, Casalini F, et al. Inhibition of metalloproteinases derived from tumours: new insights in the treatment of human glioblastoma. Neuroscience. 2010;168(2):514-22. Epub 2010/04/13. doi:

10.1016/j.neuroscience.2010.03.064. PubMed PMID: 20382206.

73. Levin VA, Phuphanich S, Yung WK, Forsyth PA, Maestro RD, Perry JR, et al.

Randomized, double-blind, placebo-controlled trial of marimastat in glioblastoma multiforme patients following surgery and irradiation. J Neurooncol. 2006;78(3):295-302. Epub 2006/04/26. doi: 10.1007/s11060-005-9098-5. PubMed PMID: 16636750.

74. Xue Q, Cao L, Chen XY, Zhao J, Gao L, Li SZ, et al. High expression of MMP9 in glioma affects cell proliferation and is associated with patient survival rates. Oncol Lett.

2017;13(3):1325-30. Epub 2017/04/30. doi: 10.3892/ol.2017.5567. PubMed PMID: 28454256; PubMed Central PMCID: PMCPMC5403257.

75. Andersen DS, Colombani J, Palmerini V, Chakrabandhu K, Boone E, Rothlisberger M, et al. The Drosophila TNF receptor Grindelwald couples loss of cell polarity and neoplastic growth. Nature. 2015;522(7557):482-6. Epub 2015/04/16. doi: 10.1038/nature14298. PubMed PMID: 25874673.

76. Moreno E, Basler K. dMyc transforms cells into super-competitors. Cell. 2004;117(1):117-29. Epub 2004/04/07. PubMed PMID: 15066287.

77. Dominguez $M$, Wasserman JD, Freeman M. Multiple functions of the EGF receptor in Drosophila eye development. Curr Biol. 1998;8(19):1039-48. Epub 1998/10/13. PubMed PMID: 9768358.

78. Furnari FB, Fenton T, Bachoo RM, Mukasa A, Stommel JM, Stegh A, et al. Malignant astrocytic glioma: genetics, biology, and paths to treatment. Genes Dev. 2007;21(21):2683710. Epub 2007/11/03. doi: 10.1101/gad.1596707. PubMed PMID: 17974913.

79. Maher EA, Furnari FB, Bachoo RM, Rowitch DH, Louis DN, Cavenee WK, et al. Malignant glioma: genetics and biology of a grave matter. Genes Dev. 2001;15(11):1311-33. Epub 2001/06/08. doi: 10.1101/gad.891601. PubMed PMID: 11390353.

80. Holland EC. Glioblastoma multiforme: the terminator. Proc Natl Acad Sci U S A. 2000;97(12):6242-4. Epub 2000/06/07. PubMed PMID: 10841526; PubMed Central PMCID: PMCPMC33993.

81. Kegelman TP, Hu B, Emdad L, Das SK, Sarkar D, Fisher PB. In vivo modeling of malignant glioma: the road to effective therapy. Adv Cancer Res. 2014;121:261-330. Epub 2014/06/04. doi: 10.1016/B978-0-12-800249-0.00007-X. PubMed PMID: 24889534.

82. Read RD. Drosophila melanogaster as a model system for human brain cancers. Glia. 2011;59(9):1364-76. Epub 2011/05/04. doi: 10.1002/glia.21148. PubMed PMID: 21538561; PubMed Central PMCID: PMCPMC3221733.

83. Read RD, Fenton TR, Gomez GG, Wykosky J, Vandenberg SR, Babic I, et al. A kinomewide RNAi screen in Drosophila Glia reveals that the RIO kinases mediate cell proliferation and survival through TORC2-Akt signaling in glioblastoma. PLoS Genet. 2013;9(2):e1003253. Epub 2013/03/06. doi: 10.1371/journal.pgen.1003253. PubMed PMID: 23459592; PubMed Central PMCID: PMCPMC3573097.

84. Brand AH, Perrimon N. Targeted gene expression as a means of altering cell fates and generating dominant phenotypes. Development. 1993;118(2):401-15. Epub 1993/06/01. PubMed PMID: 8223268.

85. Dear ML, Dani N, Parkinson W, Zhou S, Broadie K. Two classes of matrix metalloproteinases reciprocally regulate synaptogenesis. Development. 2016;143(1):75-87. 
bioRxiv preprint doi: https://doi.org/10.1101/520346; this version posted January 14,2019 . The copyright holder for this preprint (which was not certified by peer review) is the author/funder, who has granted bioRxiv a license to display the preprint in perpetuity. It is made available under aCC-BY-NC-ND 4.0 International license.

Epub 2015/11/26. doi: 10.1242/dev.124461. PubMed PMID: 26603384; PubMed Central PMCID: PMCPMC4725201. 


\section{Portela_Figure4}

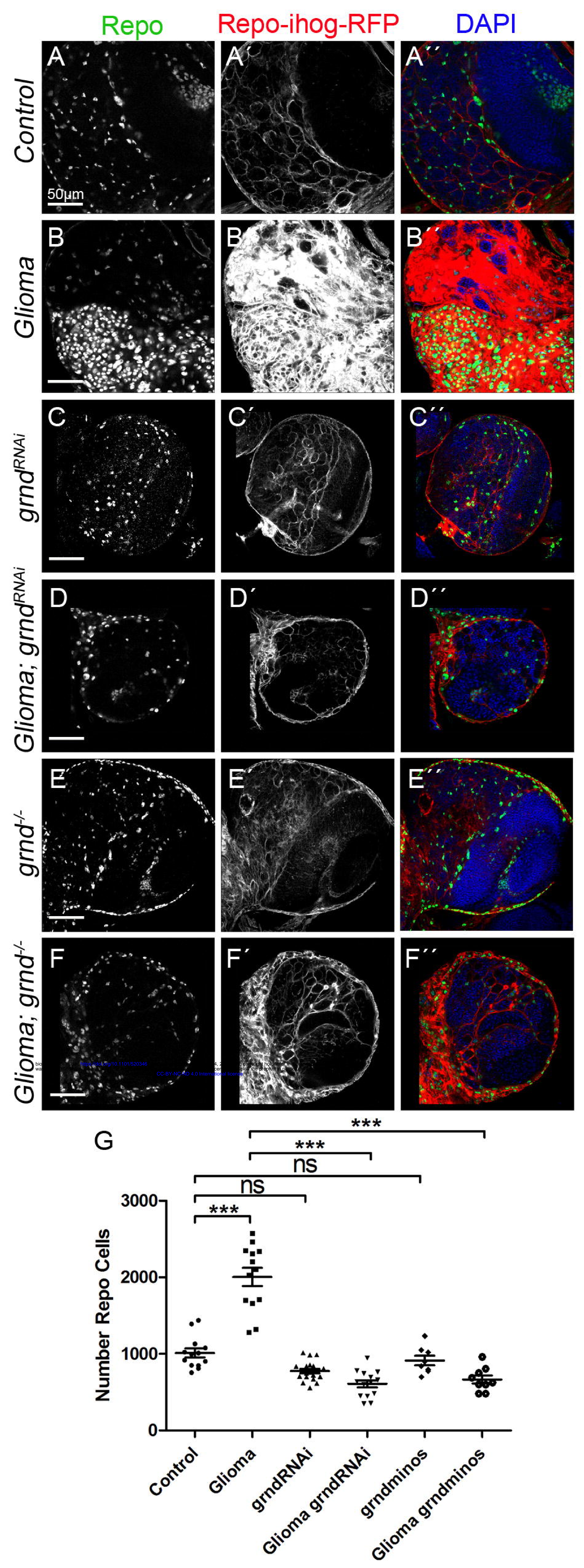


Portela_Figure5

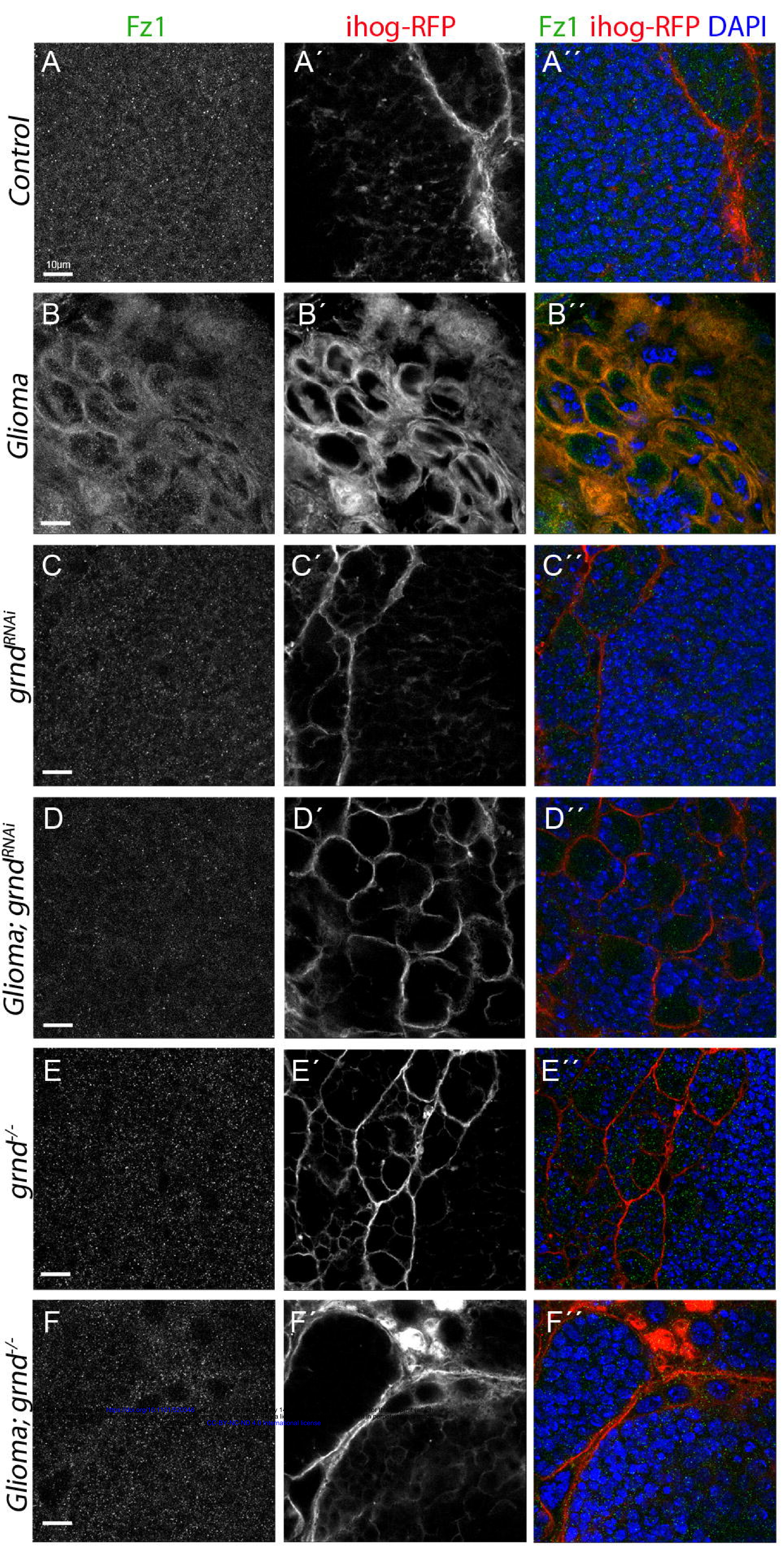

G

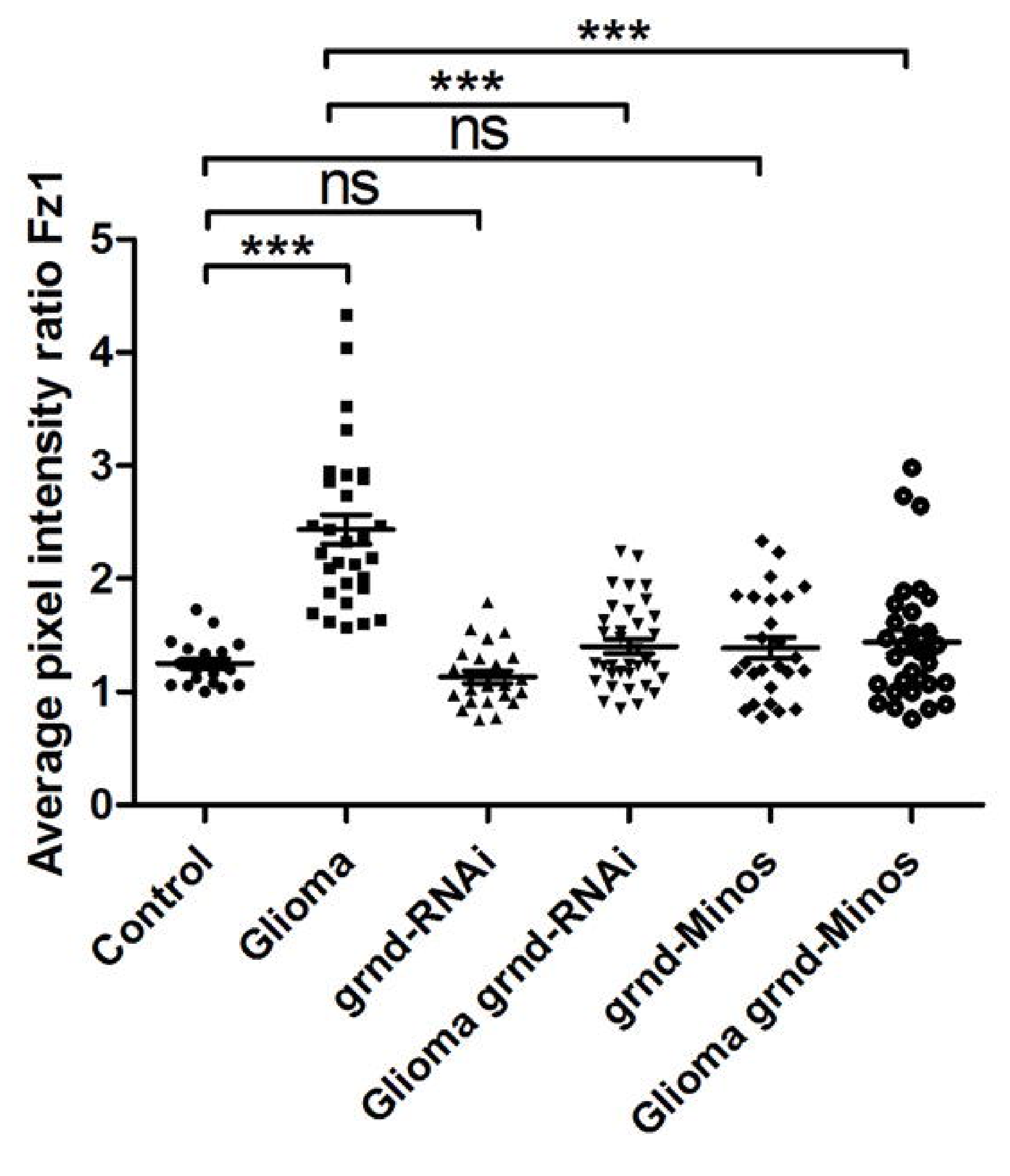




\section{Portela_Figure6}

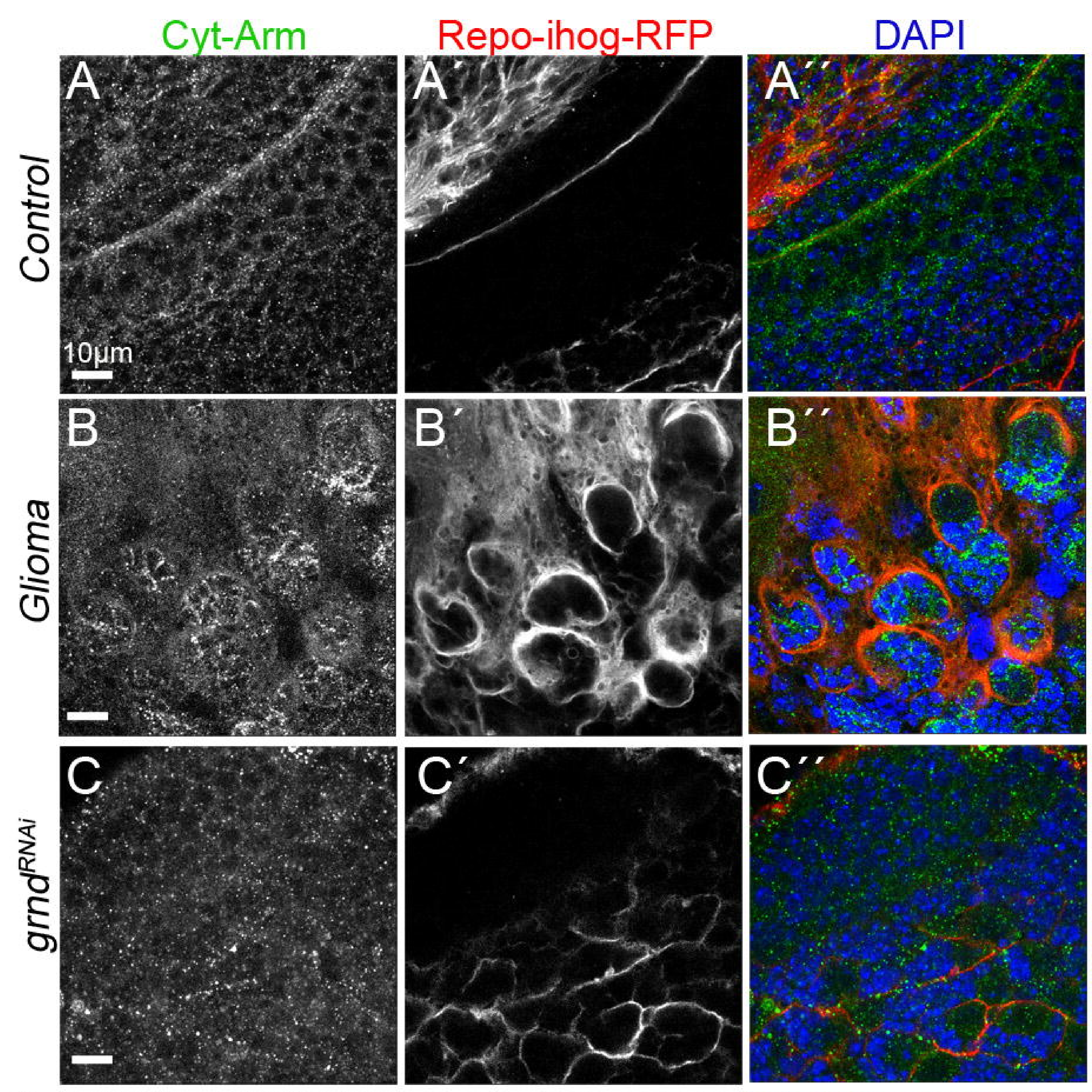

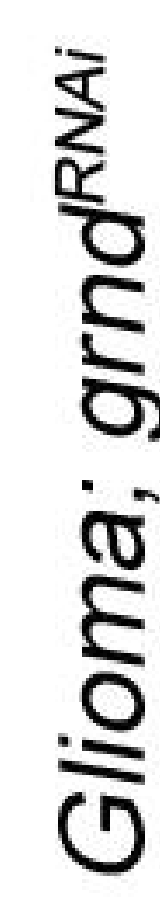

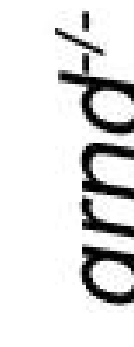

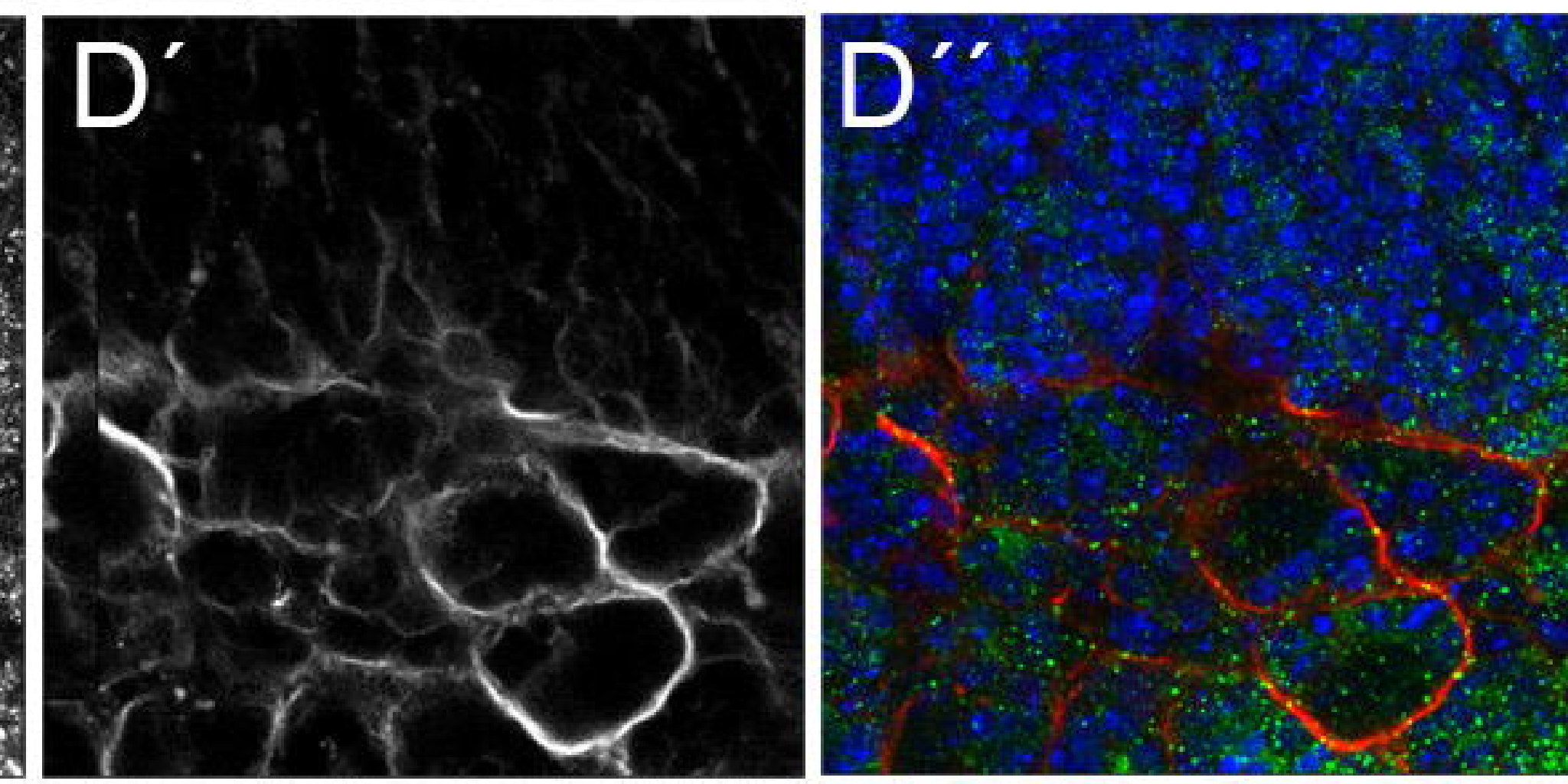

E

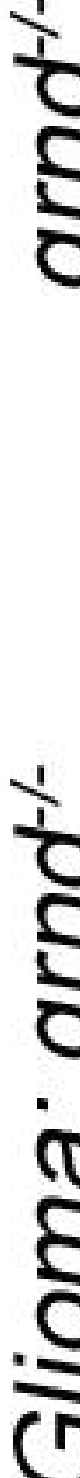

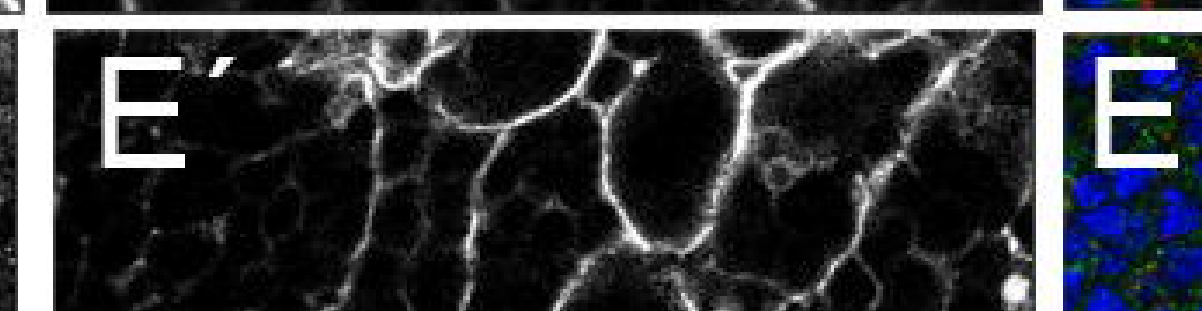

E
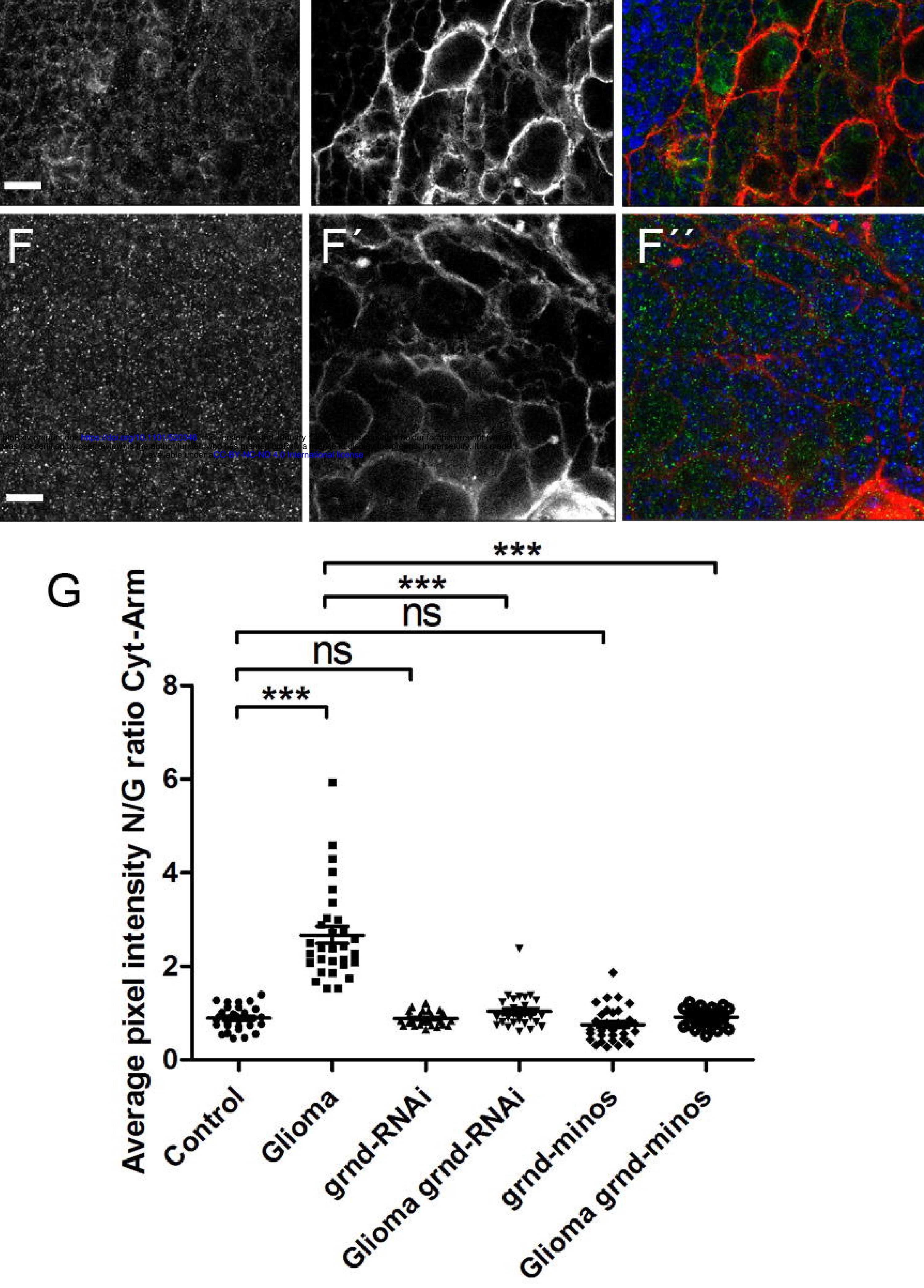


\section{Portela_Figure7}
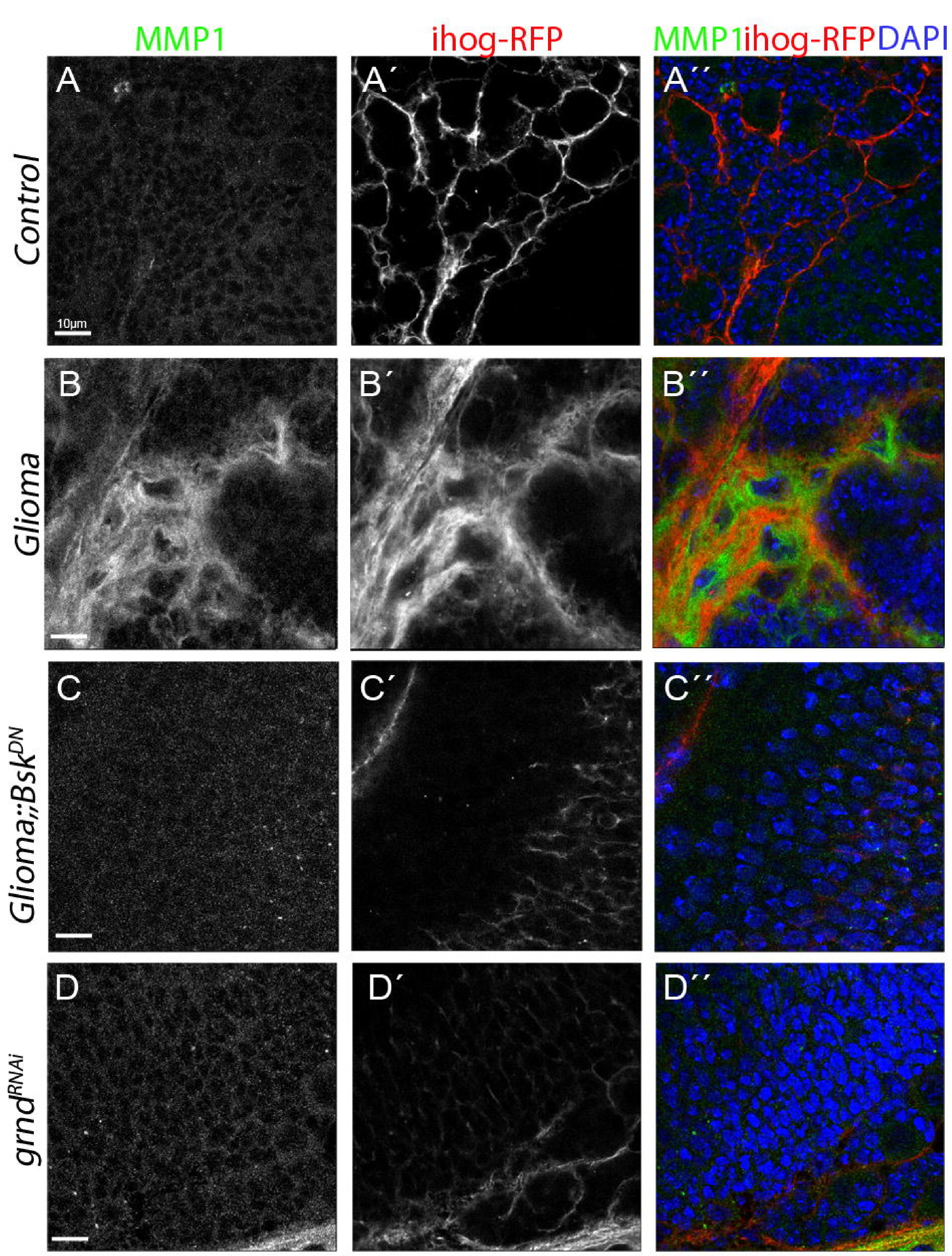

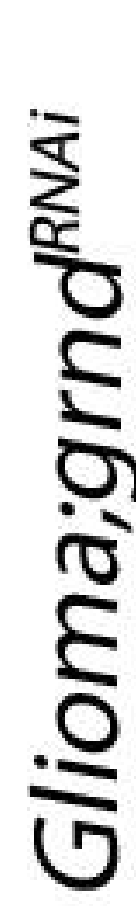
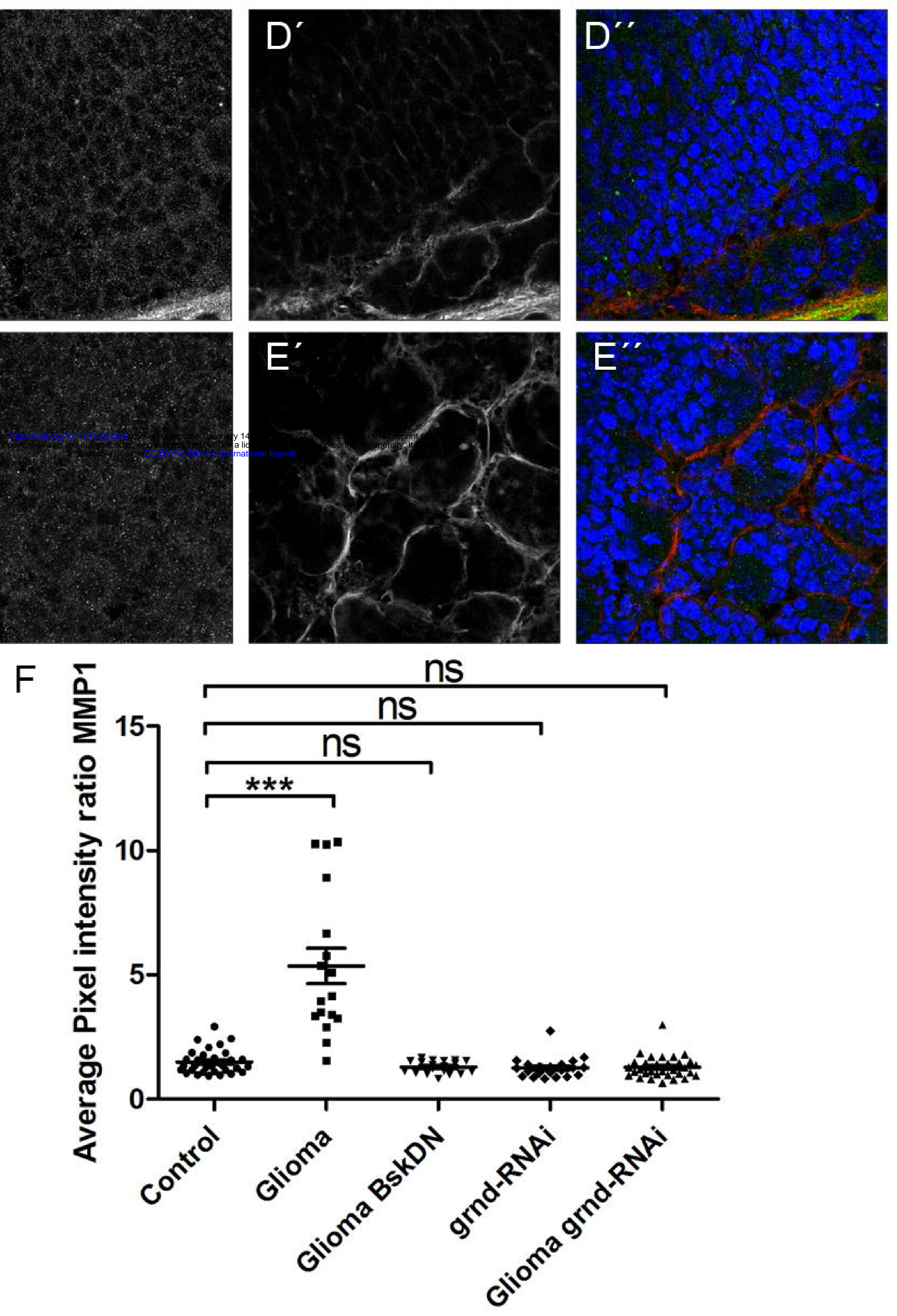

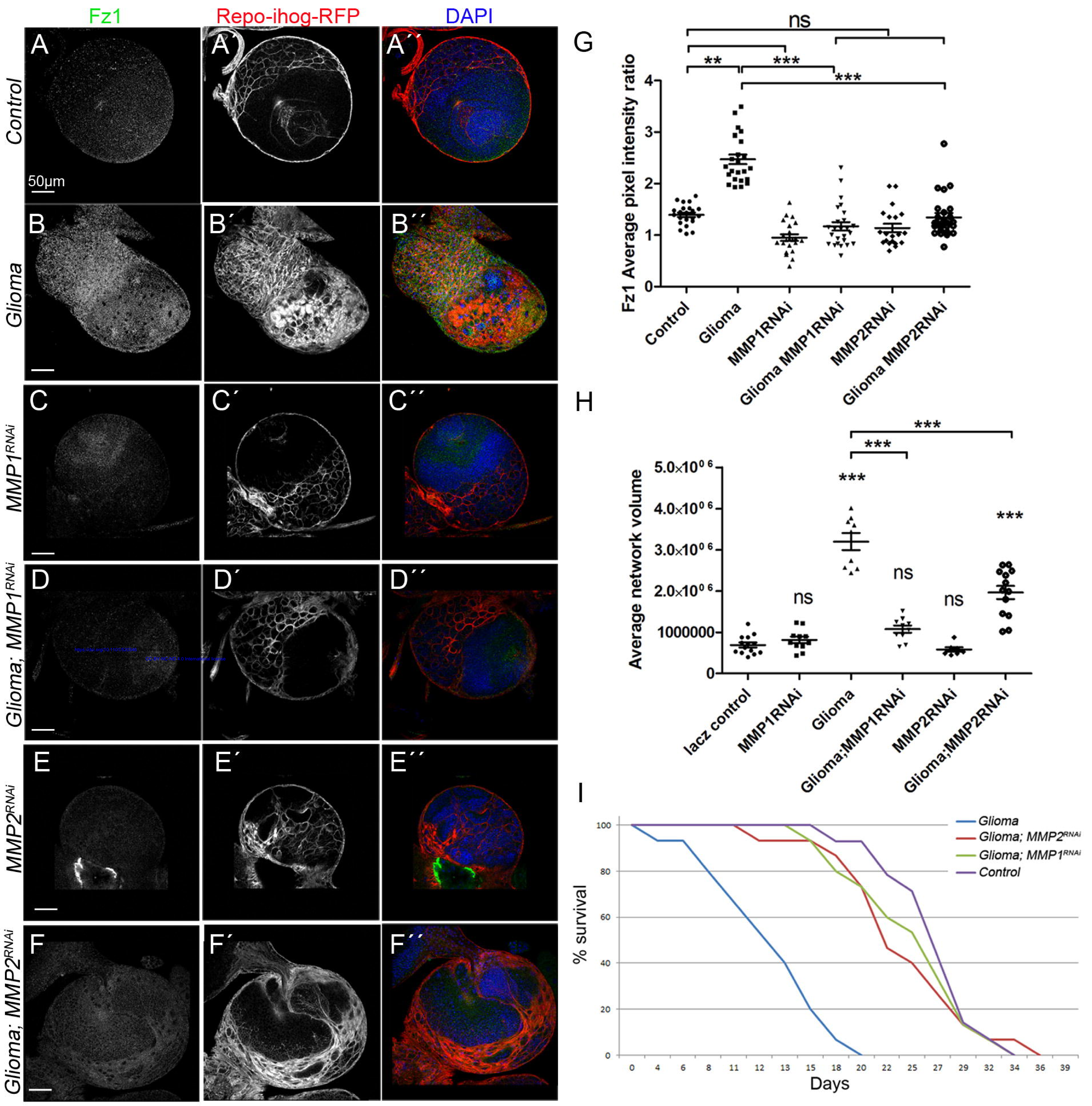
Portela_Figure9
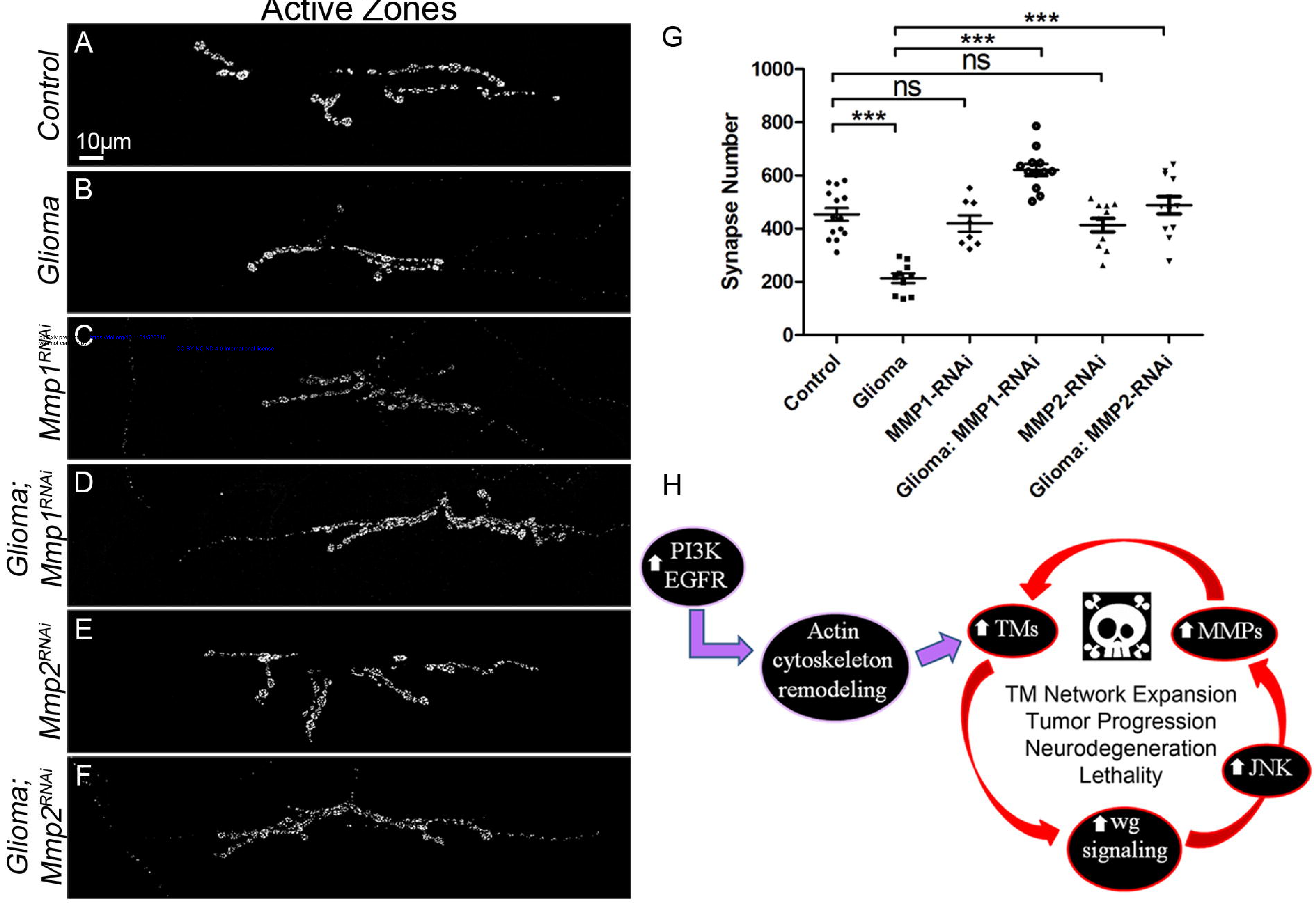